\title{
Socioeconomic and Racial/Ethnic Disparities in Cancer Mortality, Incidence, and Survival in the United States, 1950-2014: Over Six Decades of Changing Patterns and Widening Inequalities
}

\author{
Gopal K. Singh ${ }^{1}$ and Ahmedin Jemal ${ }^{2}$ \\ ${ }^{1}$ US Department of Health and Human Services, Office of Health Equity, Health Resources and Services Administration, \\ 5600 Fishers Lane, Room 13N42, Rockville, MD 20857, USA \\ ${ }^{2}$ American Cancer Society, Inc., Surveillance \& Health Services Research, 250 Williams Street NW, Corporate Center, \\ Atlanta, GA 30303, USA
}

Correspondence should be addressed to Gopal K. Singh; gsingh@hrsa.gov

Received 1 December 2016; Accepted 27 February 2017; Published 20 March 2017

Academic Editor: Habibul Ahsan

Copyright (c) 2017 Gopal K. Singh and Ahmedin Jemal. This is an open access article distributed under the Creative Commons Attribution License, which permits unrestricted use, distribution, and reproduction in any medium, provided the original work is properly cited.

\begin{abstract}
We analyzed socioeconomic and racial/ethnic disparities in US mortality, incidence, and survival rates from all-cancers combined and major cancers from 1950 to 2014. Census-based deprivation indices were linked to national mortality and cancer data for area-based socioeconomic patterns in mortality, incidence, and survival. The National Longitudinal Mortality Study was used to analyze individual-level socioeconomic and racial/ethnic patterns in mortality. Rates, risk-ratios, least squares, log-linear, and Cox regression were used to examine trends and differentials. Socioeconomic patterns in all-cancer, lung, and colorectal cancer mortality changed dramatically over time. Individuals in more deprived areas or lower education and income groups had higher mortality and incidence rates than their more affluent counterparts, with excess risk being particularly marked for lung, colorectal, cervical, stomach, and liver cancer. Education and income inequalities in mortality from all-cancers, lung, prostate, and cervical cancer increased during 1979-2011. Socioeconomic inequalities in cancer mortality widened as mortality in lower socioeconomic groups/areas declined more slowly. Mortality was higher among Blacks and lower among Asian/Pacific Islanders and Hispanics than Whites. Cancer patient survival was significantly lower in more deprived neighborhoods and among most ethnic-minority groups. Cancer mortality and incidence disparities may reflect inequalities in smoking, obesity, physical inactivity, diet, alcohol use, screening, and treatment.
\end{abstract}

\section{Introduction}

Monitoring and reducing health disparities according to socioeconomic status (SES) and race/ethnicity have long been an important health policy goal in the United States [1-3]. Studies have shown the dynamic nature of socioeconomic disparities in cancer rates as the association between SES and incidence and mortality from major cancers has changed markedly during the past 5 decades [4-7]. Temporal patterns have changed largely as a result of differential rates of decline or increase in mortality among those in various socioeconomic groups and changing sociodemographic patterns in major cancer risk factors such as smoking, diet, obesity, and physical inactivity [3-6].

Association between cancer mortality/incidence and SES varies by cancer type [3-16]. Contemporary data indicate lower rates of lung, stomach, liver, cervical, esophageal, and oropharyngeal cancer and higher rates of breast cancer and melanoma at higher SES levels [3-18]. The major behavioral determinants of cancer, such as smoking, diet, alcohol use, obesity, physical inactivity, reproductive behavior, occupational and environmental exposures, and cancer screening, 
are themselves substantially influenced by individual-level and area-level socioeconomic factors $[2,3,6,7,15,18-$ 20].

Analyzing socioeconomic and racial/ethnic patterns in cancer mortality and incidence is important because it allows us to quantify cancer-related health disparities between the least- and most-advantaged social groups and to identify areas or population groups that are at greatest risk of cancer diagnosis and mortality and who may therefore benefit from targeted social and medical interventions $[3,6]$. Temporal analyses can be used to track progress toward reducing health disparities as recent cancer disparities can be compared with those that existed in the previous decades $[3,6]$. Comparison of cancer trends across population groups or areas may provide important insights into the impact of cancer control interventions, such as smoking cessation, cancer screening, physical activity campaigns, and cancer treatment [3-7, $10]$.

Reliable individual-level SES data for all ages, especially for ages 65 and older, are lacking on US death certificates, which provide the basis for computing cancer mortality rates for various demographic groups and geographic areas [3$7,10,21]$. Individual-level data on education and income are not available for cancer patients in the Surveillance, Epidemiology, and End Results (SEER) database, which has been the primary source of data on cancer incidence, stage at diagnosis, treatment, and survival patterns in the US for the past 4 decades $[6,10,16,22]$. Because of these data limitations, population-based studies of socioeconomic disparities in cancer rates have utilized area-based socioeconomic data linked to both individual- and aggregatelevel cancer data $[3-7,10,14,17,18]$. Recent linkages of the Census and Current Population Survey (CPS) records with the National Death Index and cancer patient medical records have led to the development of longitudinal, cohort databases, allowing estimation of cancer incidence, mortality, disease stage, and survival patterns according to individual-level socioeconomic characteristics $[11,16,23$, 24].

In this paper, we extend previous analyses by examining the extent to which long-term cancer disparities in the US have changed during the last six decades. We use a census-based deprivation index to examine temporal area socioeconomic and racial/ethnic disparities in US all-cancer, lung, colorectal, prostate, breast, and cervical cancer mortality and assess racial/ethnic and area-based socioeconomic patterns in cancer incidence and survival using the SEER database. Using prospectively linked census and mortality records, we analyze temporal individual-level racial/ethnic and socioeconomic inequalities in mortality from all-cancers combined and lung, colorectal, prostate, breast, cervical, stomach, liver, and esophageal cancers. Lung cancer is the leading cause of cancer mortality, and colorectal, prostate, and breast cancers are among the most commonly diagnosed cancers; these sites, along with stomach, liver, esophageal, and cervical cancer, contribute greatly to the overall cancer burden in the US $[3,21,22,25,26]$. Taken together, these cancers account for more than half of all-cancer deaths and new cancer cases in the US $[22,25,26]$.

\section{Methods}

We examined disparities in cancer mortality, incidence, and survival using three national data sources: the national mortality database, the 1979-2011 National Longitudinal Mortality Study (NLMS), and the SEER cancer registry database $[2,3,11,21-23]$. The national mortality database has been the primary source of mortality analyses and surveillance by age, sex, race/ethnicity, cause of death, and place of residence for over a century $[2,3,6,21]$. Since the vital-statistics-based national mortality database lacks reliable socioeconomic data for all ages, socioeconomic patterns in mortality were derived by linking county-level socioeconomic data from the 1970-2000 decennial censuses with the national mortality statistics $[3-7,10,17]$. The NLMS is a longitudinal dataset for examining socioeconomic, occupational, and demographic factors associated with all-cause and cause-specific mortality in the US [23]. The NLMS is conducted by the National Heart, Lung, and Blood Institute in collaboration with the Census Bureau, the National Cancer Institute (NCI), the National Institute on Aging, and the National Center for Health Statistics. The NLMS consists of 39 CPS and census cohorts between 1973 and 2011 whose survival (mortality) experiences were studied from 1979 to 2011 [23]. The NCI's SEER database included incidence and patient survival data from 11 population-based SEER cancer registries, including the states of Connecticut, Hawaii, Iowa, New Mexico, and Utah, and metropolitan areas of Atlanta, Detroit, Los Angeles, San Francisco-Oakland, San Jose-Monterey, and Seattle [22].

We used previously developed factor-based deprivation indices from the 1970, 1990, and 2000 decennial censuses and the 2008-2012 American Community Survey to examine temporal SES disparities in cancer mortality, incidence, and survival $[3-5,27,28]$. The county deprivation index consisted of 11 census-based social indicators, viewed as broadly representing educational opportunities, labor force skills, economic and housing conditions, and general living standards in a given county $[3-5,27]$. Selected indicators of education, occupation, wealth, income distribution, unemployment rate, poverty rate, and housing quality were used to construct the index $[3-5,27]$. The neighborhood (census tract) deprivation index consisted of 17 social indicators and was used in the incidence and survival analyses. Higher index scores denote higher levels of SES and lower levels of deprivation. Details of the US deprivation indices are provided elsewhere [3-5, 27].

Index scores were categorized into 5 area groups, ranging from being the most-deprived (first quintile) to the least-disadvantaged (fifth quintile) county or neighborhood groups [3-6, 27]. The 1970 SES/deprivation index was used to calculate mortality rates from 1950 to 1974; the 1990 deprivation index was used to calculate mortality rates from 1975 to 1998; the 2000 deprivation index was used to calculate mortality rates from 1999 to 2008; and the 2008-2012 index was used to calculate mortality rates from 2009 to 2014 [35]. For census tract-level socioeconomic patterns in incidence and survival, the 1990 deprivation index was linked to the 1988-1999 incidence data from 11 SEER cancer registries [6].

Cancer mortality and incidence rates for each county, area deprivation, or individual-level socioeconomic and 


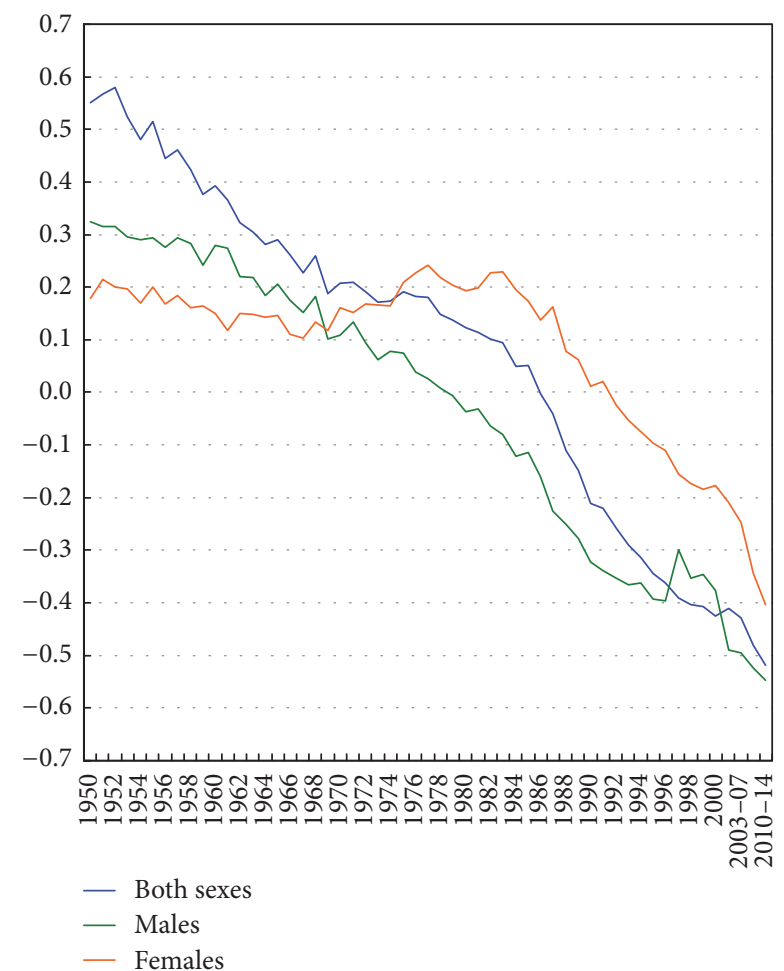

FIGURE 1: Weighted correlations between area socioeconomic index and county-level age-adjusted cancer mortality rates, United States, 1950-2014.

racial/ethnic group were age-adjusted by the direct method using the 2000 US standard population $[6,17,21,22]$. Weighted least squares regression models were fitted to county-level age-adjusted cancer mortality rates annually to estimate correlations of socioeconomic deprivation with allcancer mortality from 1950 to 2014. Log-linear regression models were used to estimate annual rates of change in SESspecific mortality trends during 1950-2013 [17, 18]. Socioeconomic disparities in mortality and incidence were described by rate ratios (relative risks) and rate differences (absolute inequalities), which were tested for statistical significance at the 0.05 level. Trend tests across SES categories were conducted using both 0.01 and 0.05 levels of significance. Unless otherwise noted, the word "significant" denotes statistically significant difference at the 0.05 level. Cause-specific survival rates were computed for men and women diagnosed with malignant cancer during 1988-1999 who were followed for vital status through December 31, 1999 [22]. Disparities in SEER-based patient survival were analyzed by multivariate Cox regression models. Cohort-based mortality rates were derived from the NLMS data by using the person-years approach $[16,23]$.

\section{Results}

3.1. Area Socioeconomic and Racial Disparities in Cancer Mortality. Figures 1 and 2 show changing socioeconomic patterns in US all-cancer mortality rates over time. The correlation between area-level SES and all-cancer mortality rates changed from +0.55 in 1950 to -0.52 in 2014 . The relationship between SES and all-cancer mortality rates reversed earlier for males than females. Between 1950 and 2014, the correlation changed from +0.33 to -0.55 for males and from +0.18 to -0.40 for females (Figure 1). Changing socioeconomic patterns in rates are more easily discernible in Figure 2, which shows a positive SES gradient in all-cancer mortality rates from 1950 through the mid-1980s and an increasingly inverse SES gradient since the mid-1990s. In 1950, those in the most-deprived group had $27 \%$ lower cancer mortality, but by $2010-2014$, they had a $22 \%$ higher cancer mortality rate compared to those in the most-affluent group. Socioeconomic gradients and absolute inequalities were steeper for men than for women. In 2010-2014, compared to their counterparts in the leastdeprived group, men had 29\% higher cancer mortality and women $15 \%$ higher mortality in the most-deprived group (Figure 2).

Long-term trends show a reversal of Black-White disparities in all-cancer mortality during 1950-2014 (Figure 3). During the early 1950s, Blacks/African Americans had lower all-cancer mortality than Whites. Since the 1960s, cancer mortality rates have been significantly higher for Blacks than for Whites. Cancer mortality rates for Blacks increased dramatically during 1950-1990. Since the early 1990s, mortality rates have declined for both Blacks and Whites (Figure 3).

Socioeconomic trends in lung cancer mortality differed for men and women. From 1950 to 1974, men in more affluent areas had higher lung cancer mortality than those in more deprived areas. Socioeconomic differentials reversed and started to widen by the early 1980s for men and by 2002 for women (Figure 4). In 2009-2013, socioeconomic inequalities in lung cancer mortality were larger and more consistent for men than for women. Men and women in the most-deprived group had $54 \%$ and $16 \%$ higher lung cancer mortality rates than their most-affluent counterparts, respectively.

During 1950-1990, lung cancer mortality among men increased at $5.1 \%$ per year in the most-deprived group, significantly faster than the annual rate of increase of $2.8 \%$ for men in the most-affluent group. Moreover, during 1991-2013, lung cancer mortality fell at a more rapid pace for men in the more affluent groups (2.53\% annually in the most-affluent group versus $1.61 \%$ in the most-deprived group). During 1950-2013, there were marked increases in lung cancer mortality among women in all deprivation groups, although the annual rate of increase in mortality was somewhat higher in the more deprived groups.

Socioeconomic trends in US colorectal cancer mortality changed dramatically between 1950 and 2013, with the positive SES gradients in mortality narrowing over time and then reversing at the turn of the 21st century (Figure 4). In 2009-2013, there was an inverse SES gradient, with those in the most-deprived group having a 30\% higher colorectal cancer mortality rate than their most-affluent counterparts. During 1950-2013, colorectal cancer mortality increased at $0.25 \%$ per year in the most-deprived group, whereas it fell consistently in the higher SES groups; the annual rates of decline in mortality in the two most-affluent groups were $1.24 \%$ and $0.87 \%$, respectively. Socioeconomic trends in 

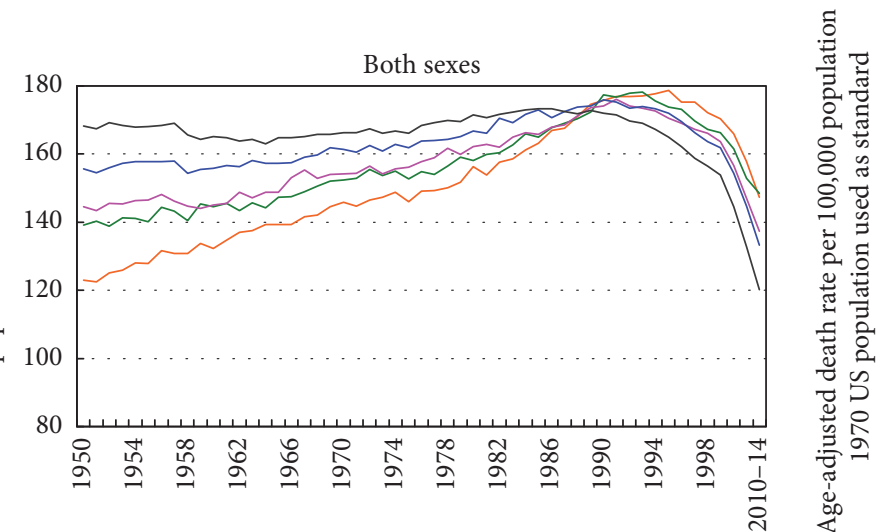

1st quintile (low SES) $\quad$ - 4th quintile
2nd quintile
3rd quintile
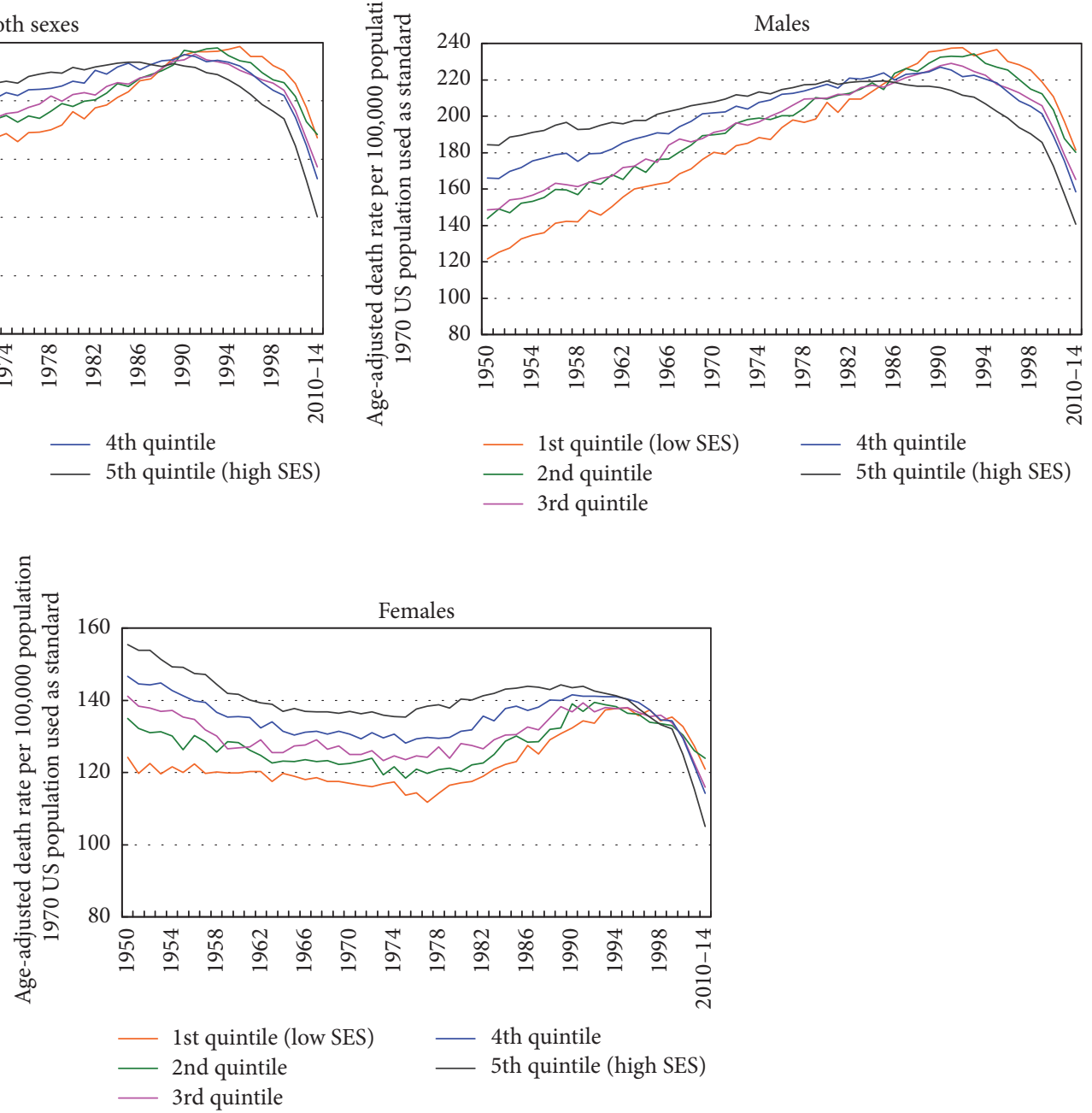

FIGURE 2: Trends in all-cancer mortality by area socioeconomic deprivation index, United States, 1950-2014.
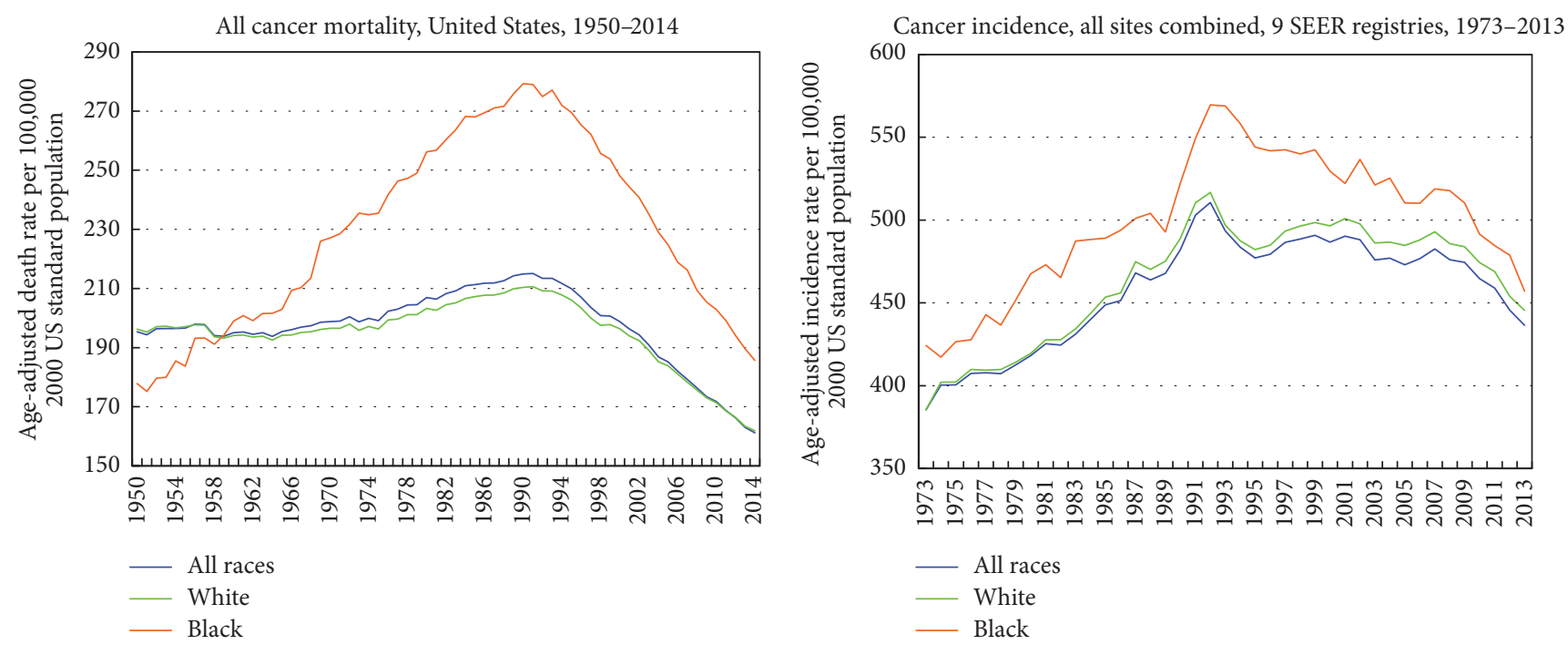

FIGURE 3: Trends in all-cancer mortality and incidence rates by race, United States, 1950-2014. The 9 SEER registries include San FranciscoOakland, Connecticut, Detroit-Metropolitan, Hawaii, Iowa, New Mexico, Seattle-Puget Sound, Utah, and Atlanta-Metropolitan. 

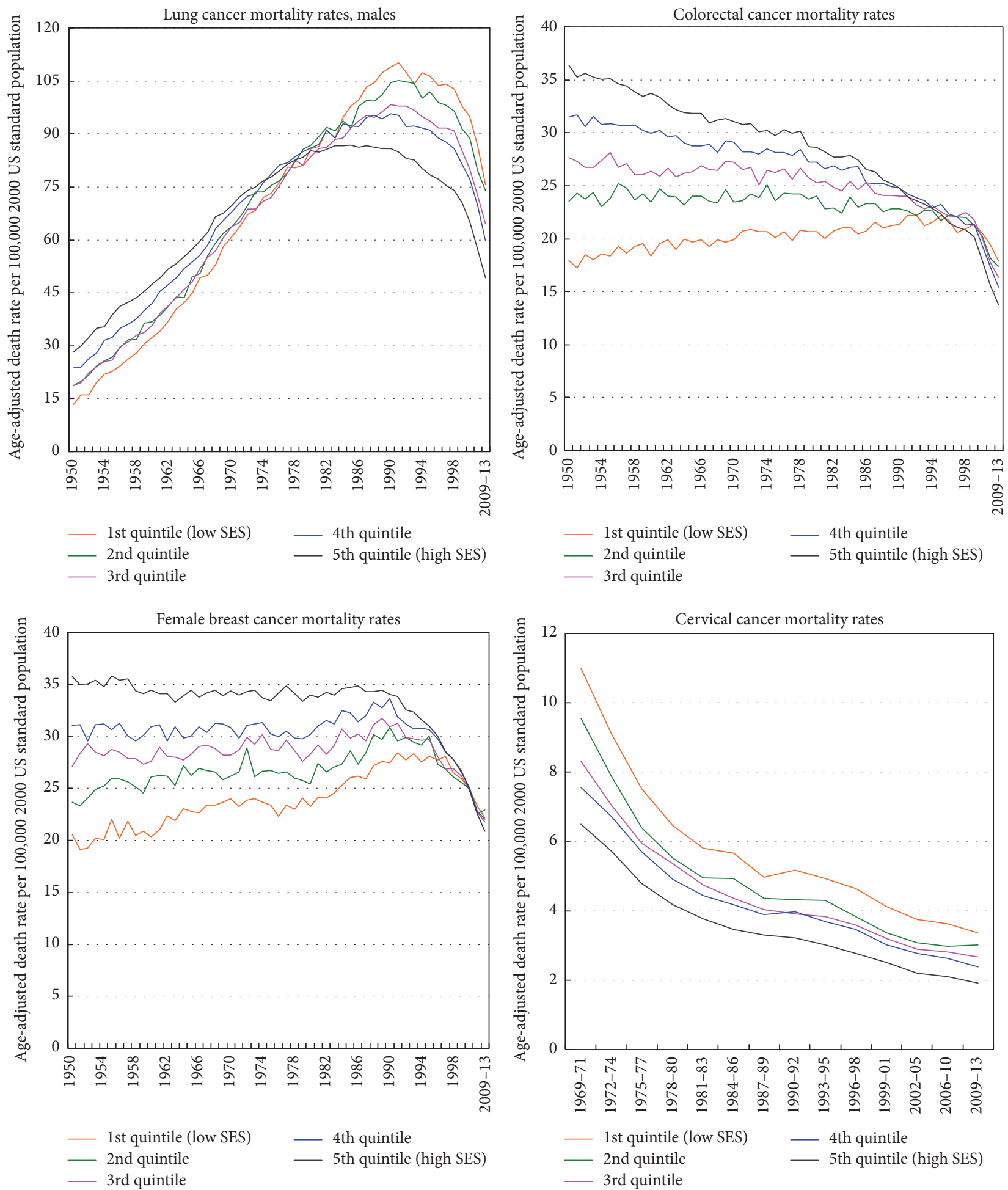

FIGURE 4: Trends in lung, colorectal, breast, and cervical cancer mortality rates by area socioeconomic deprivation index, United States, 1950-2013.

colorectal cancer mortality were generally similar for men and women.

Prostate cancer mortality did not vary appreciably over time by area deprivation. However, during the past decade, an inverse socioeconomic gradient in prostate cancer mortality was found, with mortality rates falling similarly in all deprivation groups between 1995 and 2013. In 2009-2013, men in the most-deprived group had $19 \%$ higher prostate 
cancer mortality than men in the most-affluent group (data not shown).

Socioeconomic differences in breast cancer mortality narrowed over time and reversed during the past decade, as higher deprivation levels are now associated with higher breast cancer mortality rates. The reversal of the trend has occurred as breast cancer mortality rates have declined over time for more affluent women and have increased or remained stable for women in the more deprived groups. During 1950-2013, the breast cancer mortality rate increased by $0.54 \%$ annually for women in the most-deprived group, while it decreased by $0.48 \%$ per year for women in the mostaffluent group. In 2009-2013, women in the most-deprived group had $6 \%$ higher mortality than their most-affluent counterparts. In 1950, women in the most-deprived group had $42 \%$ lower mortality than women in the most-affluent group (Figure 4).

Cervical cancer mortality rates in the US have declined consistently for the past 6 decades, and rates of mortality decline among women in all deprivation groups have been similar. However, despite the decline, substantial inverse socioeconomic gradients in cervical cancer mortality have persisted. In 2009-2013, women in the most-deprived group had a $76 \%$ higher cervical cancer mortality rate than their most-advantaged counterparts, a pattern of inequality that also characterized the trends during 1969-2008 (Figure 4).

\subsection{Individual-Level Socioeconomic and Racial/Ethnic Dispar-} ities in Cancer Mortality. All-cancer mortality rates among men varied consistently by individual-level education and income levels, with gradients in mortality being more pronounced in 2003-2011 than during 1979-1998 (Tables 1 and 2). During 2003-2011, men with less than a high school education had $68 \%$ higher cancer mortality than those with a college degree, whereas men below the poverty level had $80 \%$ higher cancer mortality than men with incomes $\geq 600 \%$ of the poverty level (Table 2). Although higher cancer mortality was associated with lower education and income levels in women, the gradients were less marked in women than in men. All-cancer mortality rates were significantly higher among Blacks and lower among Asian/Pacific Islanders (APIs) and Hispanics compared to non-Hispanic Whites.

Socioeconomic inequalities in lung cancer mortality, especially among men, were quite marked. During 2003-2011, men with less than a high school education and those below the poverty level had 2.6 times higher lung cancer mortality than their more educated and affluent counterparts. Education and income levels were also inversely related to female lung cancer mortality. Education and income inequalities in lung cancer mortality increased over time. Lung cancer mortality rates were significantly lower among APIs and Hispanics but significantly higher among Black and American Indian/Alaska Native (AIAN) men, compared to non-Hispanic Whites.

Both education and income were significantly associated with colorectal cancer mortality; men and women with less than a high school education had, respectively, $42 \%$ and $120 \%$ higher mortality risks than those with a college degree. During 2003-2011, breast cancer mortality did not vary by education and income levels. During 2003-3011, men with low education and income were at increased risk of prostate cancer mortality (Table 2). Black men had more than the risk of prostate cancer mortality than non-Hispanic White men. Prostate and breast cancer mortality was markedly lower among APIs and Hispanics compared to non-Hispanic Whites.

There were steep education and income gradients in cervical cancer mortality. During 2003-2011, women with less than a high school education and below the poverty level had 6.3 and 4.0 times higher cervical cancer mortality than women with the highest education and income levels, respectively (Table 2). Rates of stomach, liver, and esophageal cancer mortality also varied substantially and inversely by education and income levels (Table 1). Stomach and liver cancer mortality rates were much higher among APIs, Hispanics, and Blacks compared to non-Hispanic Whites.

3.3. Disparities in Site-Specific Cancer Incidence. Consistent with the mortality trends, all-cancer incidence rates showed an upward trend among both Black and White Americans until the early 1990s and have been declining since then (Figure 3). As was the case with the mortality trends, Black Americans experienced higher rates of cancer incidence than White Americans during 1973-2013.

Socioeconomic patterns in cancer incidence are generally similar to those in cancer mortality $[6,9]$. According to the 1988-1992 SEER data, higher neighborhood SES was associated with higher cancer incidence rates for the total population and for women in particular (Table 3). The male lung cancer incidence rate was $61 \%$ higher in the most-deprived than the least-deprived neighborhoods. The inverse SES gradient in male lung cancer incidence was observed for both White and Black men. Prostate cancer incidence rates increased with increasing neighborhood SES for both White and Black men. Men in the most-affluent neighborhoods had a 36\% higher prostate cancer incidence rate than men in the most-deprived neighborhoods. Higher neighborhood SES levels were associated with higher breast cancer incidence rates in both White and Black women. Women in the most-affluent neighborhoods had $47 \%$ higher breast cancer incidence rates than their most-disadvantaged counterparts. Cervical cancer incidence increased consistently with increasing deprivation levels. Women in the mostdeprived neighborhoods had a 2.7 higher risk of cervical cancer than women in the most-affluent neighborhoods. Higher deprivation levels were associated with higher rates of stomach, liver, and esophageal cancer incidence.

3.4. Disparities in Site-Specific Cancer Survival. Patient survival rates were significantly lower among men and women in more deprived neighborhoods (Figures 5 and 6). The 5year survival rate for Black patients diagnosed with cancer was $46 \%$ in the most-deprived quintile, significantly lower than the survival rate of $61 \%$ for Blacks and $66.0 \%$ for nonHispanic Whites in the least-deprived quintile. Socioeconomic gradients in cancer survival existed for all racial/ethnic groups except AIANs, with Black patients within each SES stratum experiencing lower survival than their non-Hispanic 
TABLE 1: Age-adjusted all-cancer and site-specific cancer mortality rates per 100,000 population and relative risk (RR) of mortality among those aged $\geq 25$ years by race/ethnicity, educational attainment, and poverty status: 1979-1998 National Longitudinal Mortality Study.

\begin{tabular}{|c|c|c|c|c|c|c|c|c|c|}
\hline & \multicolumn{3}{|c|}{ Age-adjusted mortality } & \multicolumn{3}{|c|}{ Age-adjusted mortality } & \multicolumn{3}{|c|}{ Age-adjusted mortality } \\
\hline & Rate & SE & $\mathrm{RR}$ & Rate & SE & $\mathrm{RR}$ & Rate & SE & $\mathrm{RR}$ \\
\hline & \multicolumn{3}{|c|}{ All cancers combined, male } & \multicolumn{3}{|c|}{ All cancers combined, female } & \multicolumn{3}{|c|}{ Lung cancer, male } \\
\hline \multicolumn{10}{|l|}{ Race/ethnicity } \\
\hline Non-Hispanic White & 355.67 & 2.53 & 1.00 & 230.37 & 1.75 & 1.00 & 116.79 & 1.42 & 1.00 \\
\hline Non-Hispanic Black & 471.66 & 10.06 & $1.33^{*}$ & 274.13 & 6.05 & $1.19^{*}$ & 146.18 & 5.50 & $1.25^{*}$ \\
\hline American Indian/Alaska Native & 334.70 & 31.37 & 0.94 & 255.35 & 22.74 & 1.11 & 119.49 & 18.55 & 1.02 \\
\hline Asian/Pacific Islander & 229.76 & 14.00 & $0.65^{*}$ & 144.98 & 10.42 & $0.63^{*}$ & 55.26 & 6.80 & $0.47^{*}$ \\
\hline Mexican American & 261.37 & 13.35 & $0.73^{*}$ & 150.75 & 8.60 & $0.65^{*}$ & 65.23 & 6.81 & $0.56^{*}$ \\
\hline Other Hispanic & 241.55 & 13.14 & $0.68^{*}$ & 141.48 & 8.14 & $0.61^{*}$ & 57.63 & 6.14 & $0.49^{*}$ \\
\hline Educational attainment (years) & \multicolumn{3}{|c|}{$\left(P_{\text {trend }}\right.$ for education $\left.<0.01\right)$} & \multicolumn{3}{|c|}{$\left(P_{\text {trend }}\right.$ for education $\left.<0.01\right)$} & \multicolumn{3}{|c|}{ ( $P_{\text {trend }}$ for education $\left.<0.01\right)$} \\
\hline$<12$ & 418.15 & 4.14 & $1.57^{*}$ & 251.18 & 3.05 & $1.23^{*}$ & 153.05 & 2.53 & $2.36^{*}$ \\
\hline 12 & 351.49 & 4.44 & $1.32^{*}$ & 228.32 & 2.59 & $1.12^{*}$ & 111.67 & 2.38 & $1.72^{*}$ \\
\hline $13-15$ & 334.57 & 6.92 & $1.26^{*}$ & 218.20 & 4.30 & 1.07 & 95.40 & 3.53 & $1.47^{*}$ \\
\hline $16+$ & 265.88 & 5.34 & 1.00 & 204.40 & 4.66 & 1.00 & 64.94 & 2.56 & $1.00^{*}$ \\
\hline Poverty status ${ }^{1}$ & \multicolumn{3}{|c|}{$\left(P_{\text {trend }}\right.$ for income $\left.<0.01\right)$} & \multicolumn{3}{|c|}{$\left(P_{\text {trend }}\right.$ for income $\left.<0.01\right)$} & \multicolumn{3}{|c|}{$\left(P_{\text {trend }}\right.$ for income $\left.<0.01\right)$} \\
\hline$<100 \%$ & 425.92 & 8.01 & $1.43^{*}$ & 264.01 & 4.60 & $1.26^{*}$ & 151.81 & 4.82 & $1.83^{*}$ \\
\hline $100-150 \%$ & 418.24 & 7.99 & $1.40^{*}$ & 245.02 & 5.06 & $1.17^{*}$ & 146.93 & 4.83 & $1.77^{*}$ \\
\hline $150-200 \%$ & 396.53 & 7.47 & $1.33^{*}$ & 235.06 & 4.93 & $1.12^{*}$ & 138.87 & 4.44 & $1.67^{*}$ \\
\hline $200-400 \%$ & 360.04 & 3.99 & $1.21^{*}$ & 225.60 & 2.74 & $1.08^{*}$ & 115.51 & 2.20 & $1.39^{*}$ \\
\hline $400-600 \%$ & 320.14 & 5.35 & 1.07 & 216.11 & 3.90 & 1.03 & 99.99 & 2.86 & $1.20^{*}$ \\
\hline \multirow[t]{2}{*}{ Above $600 \%$} & 298.19 & 6.15 & 1.00 & 208.97 & 4.60 & 1.00 & 83.04 & 3.06 & 1.00 \\
\hline & \multicolumn{3}{|c|}{ Lung cancer, female } & \multicolumn{3}{|c|}{ Colorectal cancer, male } & \multicolumn{3}{|c|}{ Colorectal cancer, female } \\
\hline \multicolumn{10}{|l|}{ Race/ethnicity } \\
\hline Non-Hispanic White & 51.68 & 0.83 & 1.00 & 38.77 & 0.84 & 1.00 & 26.67 & 0.58 & 1.00 \\
\hline Non-Hispanic Black & 56.81 & 2.76 & $1.10^{*}$ & 41.66 & 2.99 & $1.07^{*}$ & 34.35 & 2.14 & $1.29^{*}$ \\
\hline American Indian/Alaska Native & 44.15 & 9.48 & 0.85 & 29.18 & 9.64 & 0.75 & 31.98 & 8.13 & 1.20 \\
\hline Asian/Pacific Islander & 26.67 & 4.55 & $0.52^{*}$ & 29.85 & 5.05 & $0.77^{*}$ & 13.07 & 3.26 & $0.49^{*}$ \\
\hline Mexican American & 19.45 & 3.15 & $0.38^{*}$ & 24.11 & 4.00 & $0.62^{*}$ & 16.22 & 2.88 & $0.61^{*}$ \\
\hline Other Hispanic & 19.19 & 3.00 & $0.37^{*}$ & 21.32 & 3.91 & $0.55^{*}$ & 13.23 & 2.50 & $0.50^{*}$ \\
\hline Educational attainment (years) & \multicolumn{3}{|c|}{$\left(P_{\text {trend }}\right.$ for education $\left.<0.01\right)$} & \multicolumn{3}{|c|}{$\left(P_{\text {trend }}\right.$ for education $\left.<0.01\right)$} & $\left(P_{\text {trend }}\right.$ & ducatic & $0.05)$ \\
\hline$<12$ & 58.82 & 1.51 & $1.83^{*}$ & 40.81 & 1.25 & $1.53^{*}$ & 29.21 & 0.95 & 1.16 \\
\hline 12 & 52.84 & 1.22 & $1.65^{*}$ & 40.11 & 1.52 & $1.51^{*}$ & 25.91 & 0.89 & 1.03 \\
\hline $13-15$ & 46.06 & 1.98 & $1.44^{*}$ & 37.27 & 2.33 & $1.40^{*}$ & 24.45 & 1.46 & 0.97 \\
\hline $16+$ & 32.08 & 1.87 & 1.00 & 26.65 & 1.70 & 1.00 & 25.12 & 1.67 & 1.00 \\
\hline Poverty status ${ }^{1}$ & $\left(P_{\text {trer }}\right.$ & incom & $0.01)$ & $\left(P_{\text {trer }}\right.$ & incom & $0.01)$ & $\left(P_{\text {trer }}\right.$ & incom & $0.01)$ \\
\hline$<100 \%$ & 58.97 & 2.24 & $1.28^{*}$ & 39.54 & 2.41 & $1.24^{*}$ & 29.59 & 1.44 & $1.29^{*}$ \\
\hline $100-150 \%$ & 54.19 & 2.43 & $1.17^{*}$ & 41.59 & 2.47 & $1.31^{*}$ & 29.30 & 1.62 & $1.28^{*}$ \\
\hline $150-200 \%$ & 49.28 & 2.26 & 1.07 & 41.12 & 2.39 & $1.29^{*}$ & 28.20 & 1.63 & 1.23 \\
\hline $200-400 \%$ & 48.83 & 1.27 & 1.06 & 39.08 & 1.33 & $1.23^{*}$ & 25.75 & 0.93 & 1.12 \\
\hline $400-600 \%$ & 48.70 & 1.80 & 1.05 & 37.16 & 1.88 & 1.17 & 26.05 & 1.39 & 1.13 \\
\hline Above $600 \%$ & 46.18 & 2.13 & 1.00 & 31.77 & 2.05 & 1.00 & 22.96 & 1.56 & 1.00 \\
\hline & & tate can & & $\mathrm{Bre}$ & ancer, $f$ & & & ical can & \\
\hline Race/ethnicity & & & & & & & & & \\
\hline Non-Hispanic White & 44.43 & 0.93 & 1.00 & 41.72 & 0.76 & 1.00 & 3.91 & 0.24 & 1.00 \\
\hline Non-Hispanic Black & 97.31 & 4.77 & $2.19^{*}$ & 46.83 & 2.51 & $1.12^{*}$ & 8.71 & 1.08 & $2.23^{*}$ \\
\hline American Indian/Alaska Native & 43.11 & 12.00 & 0.97 & 35.79 & 8.14 & 0.86 & 6.14 & 3.09 & 1.57 \\
\hline
\end{tabular}


TABle 1: Continued.

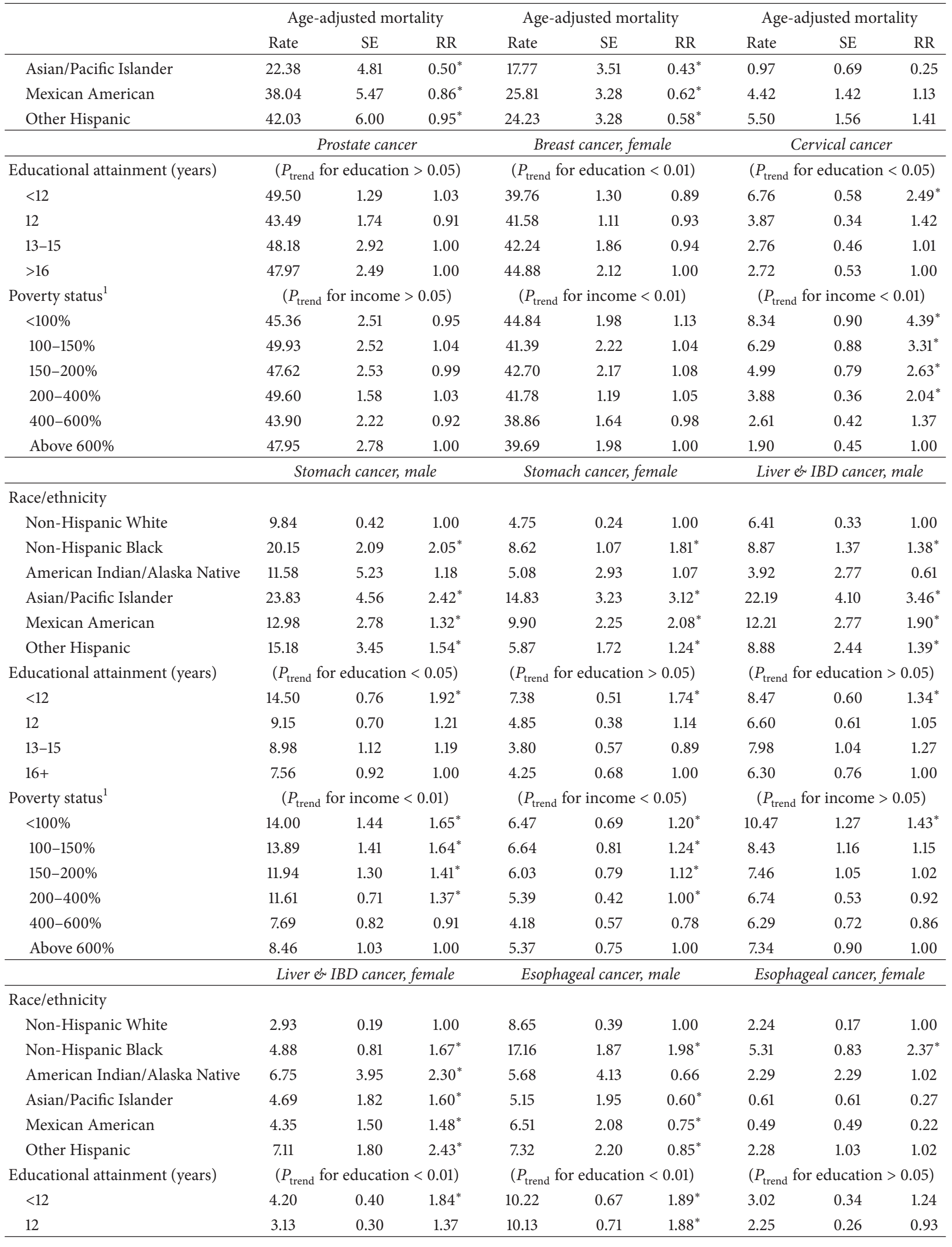


TABLE 1: Continued.

\begin{tabular}{lccccccccc}
\hline & \multicolumn{3}{c}{ Age-adjusted mortality } & \multicolumn{3}{c}{ Age-adjusted mortality } & \multicolumn{3}{c}{ Age-adjusted mortality } \\
& Rate & SE & RR & Rate & SE & RR & Rate & SE & RR \\
\hline $13-15$ & 3.08 & 0.52 & 1.35 & 8.01 & 0.98 & $1.48^{*}$ & 2.76 & 0.49 & 1.14 \\
$16+$ & 2.28 & 0.50 & 1.00 & 5.40 & 0.67 & 1.00 & 2.43 & 0.52 & 1.00 \\
Poverty status $^{1}$ & \multicolumn{2}{c}{$\left(P_{\text {trend }}\right.$} & for income $<0.01)$ & \multicolumn{2}{c}{$\left(P_{\text {trend }}\right.$ for income $\left.<0.01\right)$} & \multicolumn{2}{c}{$\left(P_{\text {trend }}\right.$ for income $\left.>0.05\right)$} \\
$<100 \%$ & 4.52 & 0.59 & $1.91^{*}$ & 13.77 & 1.48 & $1.90^{*}$ & 3.39 & 0.55 & 1.22 \\
$100-150 \%$ & 5.25 & 0.74 & $2.22^{*}$ & 9.59 & 1.27 & 1.32 & 2.67 & 0.50 & 0.96 \\
$150-200 \%$ & 3.44 & 0.58 & 1.45 & 10.72 & 1.24 & 1.48 & 3.32 & 0.60 & 1.19 \\
$200-400 \%$ & 2.77 & 0.31 & 1.17 & 8.85 & 0.61 & 1.22 & 2.12 & 0.26 & 0.76 \\
$400-600 \%$ & 2.83 & 0.45 & 1.19 & 7.42 & 0.75 & 1.02 & 2.19 & 0.40 & 0.78 \\
Above 600\% & 2.37 & 0.49 & 1.00 & 7.24 & 0.87 & 1.00 & 2.79 & 0.54 & 1.00 \\
\hline
\end{tabular}

Mortality rates are age-adjusted to the 2000 US standard population. ${ }^{*} P<0.05 .{ }^{1}$ Defined as a ratio of family income to poverty threshold.

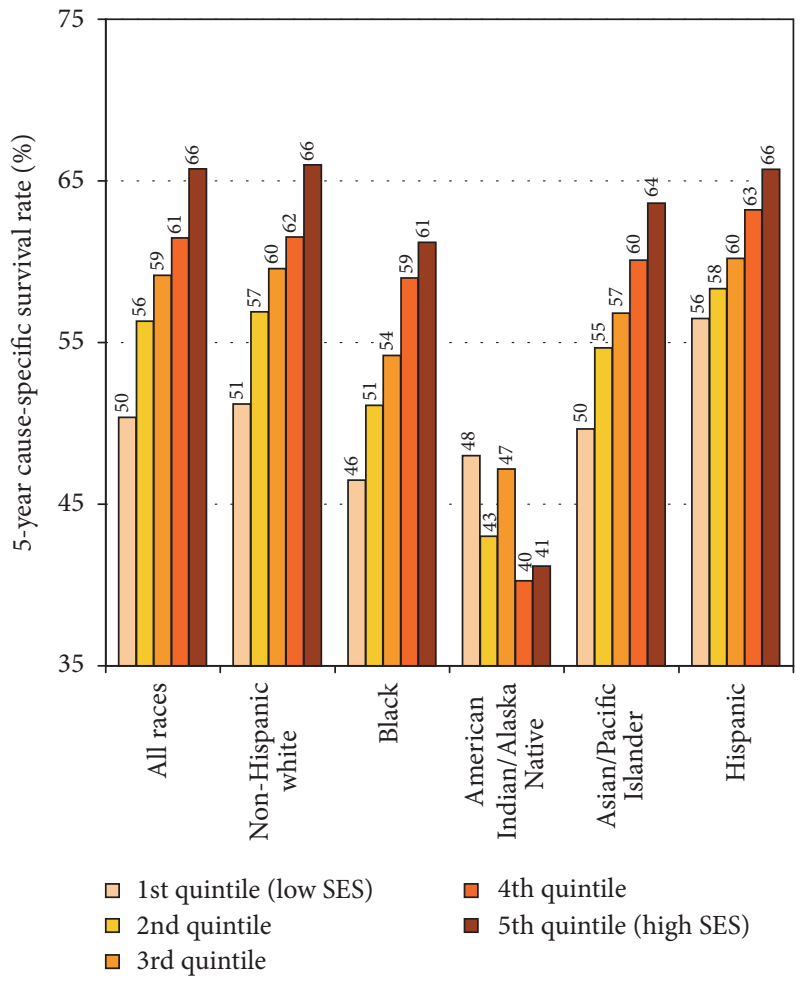

Figure 5: Cancer survival by census tract socioeconomic index and race/ethnicity, all sites and both sexes combined, 1988-94 patient cohort. Note: based on data from 11 SEER registries that include the states of Connecticut, Hawaii, Iowa, New Mexico, and Utah; and the metropolitan areas of Atlanta, Detroit, Los Angeles, San Francisco and Oakland, San Jose and Monterey, and Seattle.

White counterparts (Figure 5). Racial and SES disparities in survival existed even after controlling for stage of disease at diagnosis. Among women diagnosed with localized stage breast cancer during 1988-1994, the 5-year survival rate was $88 \%$ for Black women in the most-deprived quintile, compared with the survival rate of $91 \%$ for Black women in the least-deprived quintile; this difference in survival rates while statistically significant was relatively small (data not shown).

Differences by deprivation deciles show wide disparities in survival from all-cancers combined and colorectal, prostate, and breast cancer (Figure 6). During 1988-1999, the 10 -year survival rate for patients diagnosed with cancer was $41 \%$ in the most-deprived decile, compared with $60.4 \%$ in the least-deprived decile. The corresponding 10 -year survival rates for patients diagnosed with colorectal cancer were $49.2 \%$ and $61.5 \%$.

Table 4 shows relative risks of mortality among patients diagnosed with specific cancers during 1988-1999 after adjusting for age and period of diagnosis, sex, race/ethnicity, marital status, area deprivation, and rural-urban residence. Cancer patients in the most-deprived decile had 56\% higher adjusted risk of mortality than those in the least-deprived decile. Patient mortality by deprivation levels were particularly pronounced for breast and prostate cancer. After adjusting for deprivation and other covariates, Blacks, American Indians, and Hispanics experienced significantly higher patient mortality than non-Hispanic Whites. Several API groups such as Chinese, Filipinos, Koreans, Vietnamese, and Hawaiians had higher overall patient mortality than nonHispanic Whites.

3.5. Disparities in Risk Factors and Cancer Screening. Marked racial/ethnic and socioeconomic disparities exist in smoking, physical inactivity, dietary behavior, and cancer screening uptake (Table 5) [29]. Compared to non-Hispanic Whites, AIANs and Blacks had higher rates of smoking, obesity, and physical inactivity; APIs had lower rates of smoking and obesity; and Hispanics had higher rates of obesity. AIANs and Hispanics had lower rates of breast, cervical, and colorectal cancer screening compared to Whites. Those with lower education and income levels had significantly higher prevalence of smoking, obesity, physical inactivity, inadequate fruit/vegetable intake, and lower rates of cancer screening. 
TABLE 2: Age-adjusted all-cancer and site-specific cancer mortality rates per 100,000 population and relative risk (RR) of mortality among those aged $\geq 25$ years by race/ethnicity, educational attainment, and poverty status: 2003-2011 National Longitudinal Mortality Study.

\begin{tabular}{|c|c|c|c|c|c|c|c|c|c|}
\hline & \multicolumn{3}{|c|}{ Age-adjusted mortality } & \multicolumn{3}{|c|}{ Age-adjusted mortality } & \multicolumn{3}{|c|}{ Age-adjusted mortality } \\
\hline & Rate & SE & $\mathrm{RR}$ & Rate & SE & RR & Rate & SE & $\mathrm{RR}$ \\
\hline & \multicolumn{3}{|c|}{ All cancers combined, male } & \multicolumn{3}{|c|}{ All cancers combined, female } & \multicolumn{3}{|c|}{ Lung cancer, male } \\
\hline \multicolumn{10}{|l|}{ Race/ethnicity } \\
\hline Non-Hispanic White & 254.50 & 5.00 & 1.00 & 210.50 & 4.33 & 1.00 & 78.50 & 2.83 & 1.00 \\
\hline Non-Hispanic Black & 350.00 & 17.00 & $1.38^{*}$ & 209.50 & 11.50 & 1.00 & 114.17 & 9.83 & $1.45^{*}$ \\
\hline American Indian/Alaska Native & 406.17 & 58.50 & $1.60^{*}$ & 284.17 & 46.67 & 1.35 & 151.50 & 36.33 & $1.93^{*}$ \\
\hline Asian/Pacific Islander & 149.33 & 17.67 & $0.59^{*}$ & 159.83 & 17.33 & $0.76^{*}$ & 36.83 & 9.00 & $0.47^{*}$ \\
\hline Mexican American & 143.67 & 14.00 & $0.56^{*}$ & 127.00 & 12.67 & $0.60^{*}$ & 27.33 & 6.33 & $0.35^{*}$ \\
\hline Other Hispanic & 155.50 & 16.00 & $0.61^{*}$ & 140.67 & 13.67 & $0.67^{*}$ & 28.67 & 7.00 & $0.37^{*}$ \\
\hline Educational attainment (years) & \multicolumn{3}{|c|}{$\left(P_{\text {trend }}\right.$ for education $\left.<0.01\right)$} & \multicolumn{3}{|c|}{$\left(P_{\text {trend }}\right.$ for education $\left.<0.01\right)$} & \multicolumn{3}{|c|}{$\left(P_{\text {trend }}\right.$ for education $\left.<0.01\right)$} \\
\hline$<12$ & 287.50 & 9.33 & $1.68^{*}$ & 230.33 & 8.00 & $1.43^{*}$ & 102.83 & 5.83 & $2.57^{*}$ \\
\hline 12 & 288.33 & 8.33 & $1.68^{*}$ & 202.00 & 6.17 & $1.25^{*}$ & 89.17 & 4.67 & $2.23^{*}$ \\
\hline $13-15$ & 240.17 & 9.67 & $1.40^{*}$ & 198.33 & 8.00 & $1.23^{*}$ & 67.33 & 5.17 & $1.68^{*}$ \\
\hline $16+$ & 171.33 & 7.33 & 1.00 & 161.33 & 8.17 & 1.00 & 40.00 & 3.50 & $1.00^{*}$ \\
\hline Poverty status ${ }^{1}$ & \multicolumn{3}{|c|}{$\left(P_{\text {trend }}\right.$ for income $\left.<0.01\right)$} & \multicolumn{3}{|c|}{$\left(P_{\text {trend }}\right.$ for income $\left.<0.01\right)$} & \multicolumn{3}{|c|}{$\left(P_{\text {trend }}\right.$ for income $\left.<0.01\right)$} \\
\hline$<100 \%$ & 332.00 & 18.33 & $1.80^{*}$ & 232.83 & 11.50 & $1.55^{*}$ & 119.33 & 11.17 & $2.58^{*}$ \\
\hline $100-150 \%$ & 301.83 & 15.67 & $1.64^{*}$ & 234.00 & 11.17 & $1.55^{*}$ & 103.50 & 9.33 & $2.23^{*}$ \\
\hline $150-200 \%$ & 271.33 & 14.00 & $1.47^{*}$ & 238.83 & 11.83 & $1.59^{*}$ & 89.67 & 8.33 & $1.94^{*}$ \\
\hline $200-400 \%$ & 260.33 & 7.67 & $1.41^{*}$ & 209.17 & 6.83 & $1.39^{*}$ & 78.00 & 4.33 & $1.68^{*}$ \\
\hline $400-600 \%$ & 243.50 & 10.17 & $1.32^{*}$ & 174.33 & 9.00 & $1.16^{*}$ & 71.67 & 5.67 & $1.55^{*}$ \\
\hline \multirow[t]{2}{*}{ Above $600 \%$} & 184.17 & 8.50 & 1.00 & 150.50 & 8.33 & 1.00 & 46.33 & 4.17 & 1.00 \\
\hline & \multicolumn{3}{|c|}{ Lung cancer, female } & \multicolumn{3}{|c|}{ Colorectal cancer, male } & \multicolumn{3}{|c|}{ Colorectal cancer, female } \\
\hline \multicolumn{10}{|l|}{ Race/ethnicity } \\
\hline Non-Hispanic White & 59.83 & 2.33 & 1.00 & 23.50 & 1.50 & 1.00 & 20.00 & 1.33 & 1.00 \\
\hline Non-Hispanic Black & 52.17 & 5.83 & 0.87 & 43.33 & 6.17 & $1.84^{*}$ & 24.33 & 4.00 & 1.22 \\
\hline American Indian/Alaska Native & 54.33 & 20.50 & 0.91 & 9.00 & 9.00 & 0.38 & 40.83 & 18.17 & 2.04 \\
\hline Asian/Pacific Islander & 36.83 & 8.33 & $0.62^{*}$ & 6.50 & 3.67 & $0.28^{*}$ & 19.83 & 6.33 & 0.99 \\
\hline Mexican American & 21.00 & 5.17 & $0.35^{*}$ & 18.00 & 5.00 & 0.77 & 14.67 & 4.33 & 0.73 \\
\hline Other Hispanic & 26.67 & 6.00 & $0.45^{*}$ & 11.67 & 4.50 & 0.50 & 18.00 & 5.00 & 0.90 \\
\hline Educational attainment (years) & \multicolumn{3}{|c|}{$\left(P_{\text {trend }}\right.$ for education $\left.<0.01\right)$} & \multicolumn{3}{|c|}{$\left(P_{\text {trend }}\right.$ for education $\left.<0.01\right)$} & \multicolumn{3}{|c|}{$\left(P_{\text {trend }}\right.$ for education $\left.<0.01\right)$} \\
\hline$<12$ & 71.33 & 4.67 & $2.04^{*}$ & 26.67 & 3.00 & $1.42^{*}$ & 25.67 & 2.67 & $2.20^{*}$ \\
\hline 12 & 60.17 & 3.33 & $1.72^{*}$ & 26.83 & 2.67 & $1.42^{*}$ & 19.67 & 2.00 & $1.69^{*}$ \\
\hline $13-15$ & 41.33 & 3.67 & $1.18^{*}$ & 21.50 & 3.00 & 1.14 & 21.50 & 2.67 & $1.84^{*}$ \\
\hline $16+$ & 35.00 & 3.83 & $1.00^{*}$ & 18.83 & 2.50 & 1.00 & 11.67 & 2.17 & 1.00 \\
\hline Poverty status ${ }^{1}$ & $\left(P_{\text {trend }}\right.$ & income & $.01)$ & $\left(P_{\text {tren }}\right.$ & income & $0.05)$ & $\left(P_{\text {tren }}\right.$ & incom & $.01)$ \\
\hline$<100 \%$ & 67.83 & 6.33 & $2.30^{*}$ & 20.00 & 4.67 & 1.02 & 22.83 & 3.67 & 1.47 \\
\hline $100-150 \%$ & 68.33 & 6.17 & $2.32^{*}$ & 27.17 & 4.83 & 1.38 & 27.50 & 3.83 & $1.77^{*}$ \\
\hline $150-200 \%$ & 69.33 & 6.50 & 2.35 & 28.33 & 4.67 & 1.44 & 20.83 & 3.50 & 1.34 \\
\hline $200-400 \%$ & 57.17 & 3.50 & 1.94 & 25.33 & 2.50 & 1.29 & 23.33 & 2.33 & $1.51^{*}$ \\
\hline $400-600 \%$ & 48.50 & 4.67 & 1.64 & 24.17 & 3.17 & 1.23 & 12.00 & 2.50 & 0.77 \\
\hline Above $600 \%$ & 29.50 & 3.67 & 1.00 & 19.67 & 2.83 & 1.00 & 15.50 & 2.83 & 1.00 \\
\hline & & state car & & Brea & ancer, fe & & & cal can & \\
\hline Race/ethnicity & & & & & & & & & \\
\hline Non-Hispanic White & 23.50 & 1.50 & 1.00 & 31.67 & 1.67 & 1.00 & 1.67 & 0.33 & 1.00 \\
\hline Non-Hispanic Black & 54.00 & 6.83 & $2.30^{*}$ & 35.33 & 4.83 & 1.12 & 2.67 & 1.33 & 1.60 \\
\hline
\end{tabular}


TABLE 2: Continued.

\begin{tabular}{|c|c|c|c|c|c|c|c|c|c|}
\hline & \multicolumn{3}{|c|}{ Age-adjusted mortality } & \multicolumn{3}{|c|}{ Age-adjusted mortality } & \multicolumn{3}{|c|}{ Age-adjusted mortality } \\
\hline & Rate & SE & $\mathrm{RR}$ & Rate & SE & $\mathrm{RR}$ & Rate & SE & $\mathrm{RR}$ \\
\hline American Indian/Alaska Native & 41.83 & 20.83 & 1.78 & 38.50 & 17.17 & 1.22 & 13.33 & 9.50 & $8.00^{*}$ \\
\hline Asian/Pacific Islander & 6.67 & 3.83 & $0.28^{*}$ & 11.50 & 4.67 & $0.36^{*}$ & 1.83 & 1.83 & 1.10 \\
\hline Mexican American & 8.33 & 3.83 & $0.35^{*}$ & 16.17 & 4.50 & $0.51^{*}$ & 2.83 & 1.67 & 1.70 \\
\hline Other Hispanic & 20.67 & 6.17 & 0.88 & 17.33 & 4.83 & $0.55^{*}$ & 5.83 & 2.67 & $3.50^{*}$ \\
\hline Educational attainment (years) & \multicolumn{3}{|c|}{$\left(P_{\text {trend }}\right.$ for education $\left.>0.05\right)$} & \multicolumn{3}{|c|}{$\left(P_{\text {trend }}\right.$ for education $\left.<0.05\right)$} & \multicolumn{3}{|c|}{$\left(P_{\text {trend }}\right.$ for education $\left.<0.01\right)$} \\
\hline$<12$ & 29.33 & 2.83 & $1.63^{*}$ & 26.17 & 2.67 & 0.82 & 4.17 & 1.33 & $6.25^{*}$ \\
\hline 12 & 23.33 & 2.50 & 1.30 & 29.00 & 2.33 & 0.91 & 3.00 & 0.83 & $4.50^{*}$ \\
\hline $13-15$ & 28.00 & 3.50 & $1.56^{*}$ & 32.83 & 3.17 & 1.03 & 1.33 & 0.67 & 2.00 \\
\hline$>16$ & 18.00 & 2.50 & 1.00 & 32.00 & 3.50 & 1.00 & 0.67 & 0.33 & 1.00 \\
\hline Poverty status ${ }^{1}$ & \multicolumn{3}{|c|}{$\left(P_{\text {trend }}\right.$ for income $\left.<0.01\right)$} & \multicolumn{3}{|c|}{$\left(P_{\text {trend }}\right.$ for income $\left.>0.05\right)$} & \multicolumn{3}{|c|}{$\left(P_{\text {trend }}\right.$ for income $\left.<0.01\right)$} \\
\hline$<100 \%$ & 31.50 & 5.67 & $1.72^{*}$ & 29.00 & 4.17 & 0.90 & 4.00 & 1.67 & $4.00^{*}$ \\
\hline $100-150 \%$ & 27.17 & 4.33 & 1.48 & 28.83 & 4.00 & 0.90 & 4.00 & 1.83 & $4.00^{*}$ \\
\hline $150-200 \%$ & 26.50 & 4.00 & 1.45 & 31.00 & 4.33 & 0.96 & 4.83 & 2.00 & $4.83^{*}$ \\
\hline $200-400 \%$ & 24.50 & 2.33 & 1.34 & 31.17 & 2.67 & 0.97 & 2.83 & 0.83 & 2.83 \\
\hline $400-600 \%$ & 27.33 & 3.67 & 1.49 & 25.83 & 3.50 & 0.80 & & & \\
\hline Above $600 \%$ & 18.33 & 3.17 & 1.00 & 32.17 & 3.83 & 1.00 & 1.00 & 0.50 & 1.00 \\
\hline
\end{tabular}

${ }^{*} P<0.05 .{ }^{1}$ Defined as a ratio of family income to poverty threshold.

\section{Discussion}

In this study, we have presented a comprehensive analysis of social inequalities in cancer mortality, incidence, and patient survival from all-cancers combined and from major cancers. New analyses of area- and individual-level socioeconomic disparities in cancer mortality spanning over 6 decades are a particularly novel feature of the study. Analysis of long-term trends and contemporary socioeconomic and racial/ethnic inequalities in cancer adds to the extensive literature on cancer disparities. Socioeconomic and racial patterns in US cancer mortality have reversed over time, and the continued widening of the inverse socioeconomic gradients in allcancer, lung, and colorectal cancer mortality is consistent with those observed for all-cause and cardiovascular-disease mortality in the US $[2,3,27]$.

Consistent with past research, socioeconomic inequalities in cancer incidence and mortality in the US are particularly marked in lung, cervical, stomach, and liver cancer [3-6, $10,11,16]$. Substantial socioeconomic disparities exist not only in cancer incidence and mortality but also in stage at cancer diagnosis and survival $[3,6,10,16,24]$. Such inequalities have been shown to exist for Whites, Blacks, Hispanics, APIs, and AIANs $[3,6,10]$. Incidence and survival analyses pertain to the 1988-1999 period and serve as important benchmarks. However, these analyses need to be updated with more recent data to see if area-based socioeconomic disparities in incidence and survival have persisted or widened over time.

Area-level SES patterns in cancer incidence were generally similar to individual-level patterns derived from the linked SEER-NLMS data [16]. In the linked data, men and women with less than a high school education had 3.0 and 2.0 times higher lung cancer incidence rates, respectively, than those with a college degree [16]. Those below the poverty level had $52-72 \%$ higher lung cancer incidence rates than their counterparts with incomes at $\geq 600 \%$ of the poverty level [16]. Individuals with the lowest education and income levels had higher colorectal cancer incidence rates than their mostadvantaged counterparts [16]. Higher education and income levels were associated with higher prostate and breast cancer incidence rates [16]. Consistent with the neighborhood level pattern, women with less than a high school education had 3.2 times higher cervical cancer incidence than those with a college degree [16].

Disparities in incidence and mortality from various cancers may reflect differences in smoking prevalence, dietary patterns, obesity, physical inactivity, reproductive factors (e.g., delayed childbearing, childlessness, and breastfeeding), alcohol use, human papillomavirus (HPV) infection, cancer screening, and healthcare factors $[3,5,6,12,15,20,30]$. Higher smoking rates are more prevalent among men and women in lower SES groups and in more deprived areas (Table 5) $[2,5,17,20]$. Smoking rates have fallen more rapidly for those in higher SES groups, which largely explains temporal SES trends in all-cancer and lung cancer mortality rates $[2,4-6]$. Dietary factors such as fat intake, red meat consumption, and high calorie intake have been mentioned as risk factors for colorectal, prostate, and breast cancer and inequalities in both incidence and mortality may in part reflect differences in these factors $[3,5,6,15]$. Previous studies as well as data in Table 5 show higher consumption of lowerquality diets and energy-dense foods and lower intakes of fruits and vegetables among lower SES groups but higher total calorie and fat intake among higher SES groups $[2,3,19]$.

Healthcare disparities play a prominent role in producing socioeconomic inequalities in mortality from colorectal, 
TABle 3: Age-adjusted incidence rates per 100,000 population for all cancers combined and site-specific cancers by neighborhood (census tract) socioeconomic status (SES) index and race/ethnicity, 1988-1992, 11 SEER registries.

\begin{tabular}{|c|c|c|c|c|c|c|c|c|c|c|c|c|}
\hline SES index & $\begin{array}{c}\text { Male } \\
\text { All races } \\
\text { Rate }\end{array}$ & $\begin{array}{c}\text { Male } \\
\text { All races } \\
\text { SE }\end{array}$ & $\begin{array}{l}\text { Male } \\
\text { White } \\
\text { Rate }\end{array}$ & $\begin{array}{c}\text { Male } \\
\text { White } \\
\text { SE }\end{array}$ & $\begin{array}{l}\text { Male } \\
\text { Black } \\
\text { Rate }\end{array}$ & $\begin{array}{c}\text { Male } \\
\text { Black } \\
\text { SE }\end{array}$ & $\begin{array}{c}\text { Female } \\
\text { All races } \\
\text { Rate }\end{array}$ & $\begin{array}{l}\text { Female } \\
\text { All races } \\
\text { SE }\end{array}$ & $\begin{array}{c}\text { Female } \\
\text { White } \\
\text { Rate }\end{array}$ & $\begin{array}{c}\text { Female } \\
\text { White } \\
\text { SE }\end{array}$ & $\begin{array}{c}\text { Female } \\
\text { Black } \\
\text { Rate }\end{array}$ & $\begin{array}{c}\text { Female } \\
\text { Black } \\
\text { SE }\end{array}$ \\
\hline \multicolumn{13}{|l|}{$\begin{array}{l}\text { All cancers } \\
\text { combined }\end{array}$} \\
\hline 1st quintile & 585.07 & 2.54 & 555.65 & 3.18 & 732.34 & 5.40 & 377.44 & 1.72 & 384.31 & 2.26 & 401.44 & 3.20 \\
\hline 2nd quintile & 576.15 & 2.20 & 586.13 & 2.42 & 681.48 & 9.40 & 393.98 & 1.58 & 403.35 & 1.77 & 392.03 & 5.48 \\
\hline 3rd quintile & 595.77 & 2.19 & 607.17 & 2.36 & 714.31 & 13.13 & 409.05 & 1.53 & 417.93 & 1.67 & 404.77 & 7.44 \\
\hline 4th quintile & 594.28 & 2.21 & 602.34 & 2.32 & 659.73 & 16.70 & 417.06 & 1.54 & 424.35 & 1.63 & 418.02 & 9.95 \\
\hline 5th quintile & 588.38 & 2.22 & 595.95 & 2.33 & 650.70 & 20.04 & 431.28 & 1.58 & 438.21 & 1.67 & 414.62 & 12.58 \\
\hline RR (Q1/Q5) & 0.99 & & $0.93^{*}$ & & $1.13^{*}$ & & $0.88^{*}$ & & $0.88^{*}$ & & 0.97 & \\
\hline$P_{\text {trend }}$ & $>0.05$ & & 0.05 & & $<0.01$ & & $<0.01$ & & $<0.01$ & & $<0.05$ & \\
\hline \multicolumn{13}{|l|}{ Lung cancer } \\
\hline 1st quintile & 115.31 & 1.11 & 105.40 & 1.38 & 156.51 & 2.43 & 47.86 & 0.61 & 47.73 & 0.80 & 55.35 & 1.18 \\
\hline 2nd quintile & 101.20 & 0.92 & 101.05 & 1.00 & 137.38 & 4.13 & 45.19 & 0.53 & 46.58 & 0.60 & 48.98 & 1.92 \\
\hline 3rd quintile & 98.81 & 0.88 & 101.15 & 0.95 & 118.06 & 5.07 & 48.69 & 0.53 & 50.75 & 0.58 & 47.39 & 2.56 \\
\hline 4th quintile & 92.25 & 0.86 & 93.65 & 0.91 & 105.23 & 6.21 & 49.12 & 0.53 & 50.61 & 0.56 & 49.05 & 3.42 \\
\hline 5th quintile & 71.80 & 0.77 & 72.53 & 0.81 & 85.07 & 6.89 & 45.35 & 0.51 & 46.93 & 0.54 & 43.85 & 4.30 \\
\hline RR (Q1/Q5) & $1.61^{*}$ & & $1.45^{*}$ & & $1.84^{*}$ & & $1.06^{*}$ & & 1.02 & & $1.26^{*}$ & \\
\hline$P_{\text {trend }}$ & $<0.01$ & & $<0.01$ & & $<0.01$ & & $>0.05$ & & $>0.05$ & & $<0.01$ & \\
\hline \multicolumn{13}{|l|}{$\begin{array}{l}\text { Colorectal } \\
\text { cancer }\end{array}$} \\
\hline 1st quintile & 66.03 & 0.87 & 63.73 & 1.10 & 77.24 & 1.79 & 47.46 & 0.60 & 44.60 & 0.75 & 56.35 & 1.20 \\
\hline 2nd quintile & 72.64 & 0.79 & 74.28 & 0.87 & 72.75 & 3.10 & 50.68 & 0.55 & 50.69 & 0.60 & 59.11 & 2.20 \\
\hline 3rd quintile & 73.00 & 0.78 & 72.72 & 0.83 & 89.14 & 4.73 & 50.15 & 0.53 & 50.13 & 0.56 & 59.76 & 3.00 \\
\hline 4th quintile & 73.41 & 0.80 & 73.86 & 0.83 & 75.50 & 5.90 & 49.76 & 0.53 & 49.62 & 0.55 & 63.53 & 4.07 \\
\hline 5th quintile & 70.84 & 0.80 & 71.03 & 0.83 & 64.51 & 6.37 & 49.35 & 0.54 & 49.03 & 0.57 & 60.84 & 5.05 \\
\hline RR (Q1/Q5) & $0.93^{*}$ & & $0.90^{*}$ & & 1.20 & & $0.96^{*}$ & & $0.91^{*}$ & & 0.93 & \\
\hline$P_{\text {trend }}$ & $>0.05$ & & $>0.05$ & & $>0.05$ & & $>0.05$ & & $>0.05$ & & $<0.01$ & \\
\hline \multicolumn{13}{|c|}{ Stomach cancer } \\
\hline 1st quintile & 20.98 & 0.49 & 18.23 & 0.58 & 24.91 & 1.01 & 9.73 & 0.27 & 8.57 & 0.33 & 10.37 & 0.52 \\
\hline 2nd quintile & 13.95 & 0.35 & 12.33 & 0.36 & 21.03 & 1.70 & 6.45 & 0.20 & 5.46 & 0.20 & 9.68 & 0.90 \\
\hline 3rd quintile & 14.35 & 0.35 & 13.01 & 0.35 & 21.68 & 2.36 & 6.28 & 0.19 & 5.48 & 0.18 & 10.74 & 1.34 \\
\hline 4th quintile & 13.60 & 0.35 & 12.68 & 0.35 & 16.25 & 2.76 & 6.10 & 0.18 & 5.48 & 0.18 & 10.99 & 1.71 \\
\hline 5th quintile & 12.94 & 0.34 & 11.64 & 0.34 & 20.27 & 3.65 & 5.75 & 0.19 & 4.98 & 0.18 & 9.64 & 2.11 \\
\hline RR (Q1/Q5) & $1.62^{*}$ & & $1.57^{*}$ & & 1.23 & & $1.69^{*}$ & & $1.72^{*}$ & & 1.08 & \\
\hline$P_{\text {trend }}$ & $<0.05$ & & $<0.05$ & & $>0.05$ & & $<0.05$ & & $<0.05$ & & $>0.05$ & \\
\hline \multicolumn{13}{|c|}{$\begin{array}{l}\text { Liver and } \\
\text { intrahepatic bile } \\
\text { duct cancer }\end{array}$} \\
\hline 1st quintile & 10.39 & 0.33 & 7.78 & 0.38 & 9.52 & 0.59 & 3.37 & 0.16 & 2.66 & 0.19 & 3.33 & 0.29 \\
\hline 2nd quintile & 6.35 & 0.23 & 4.96 & 0.22 & 8.98 & 1.03 & 2.45 & 0.12 & 2.16 & 0.13 & 2.98 & 0.51 \\
\hline 3rd quintile & 5.73 & 0.21 & 4.78 & 0.21 & 7.22 & 1.24 & 2.30 & 0.11 & 1.83 & 0.11 & 2.45 & 0.58 \\
\hline 4th quintile & 5.07 & 0.20 & 4.43 & 0.20 & 6.21 & 1.39 & 1.96 & 0.10 & 1.74 & 0.10 & 3.94 & 1.02 \\
\hline 5th quintile & 5.02 & 0.20 & 3.98 & 0.19 & 8.18 & 2.37 & 2.04 & 0.11 & 1.75 & 0.11 & 0.84 & 0.63 \\
\hline RR (Q1/Q5) & $2.07^{*}$ & & $1.95^{*}$ & & 1.16 & & $1.65^{*}$ & & $1.52^{*}$ & & 3.96 & \\
\hline$P_{\text {trend }}$ & $<0.01$ & & $<0.01$ & & $>0.05$ & & $<0.01$ & & $<0.01$ & & $>0.05$ & \\
\hline
\end{tabular}


TABLE 3: Continued.

\begin{tabular}{|c|c|c|c|c|c|c|c|c|c|c|c|c|}
\hline SES index & $\begin{array}{c}\text { Male } \\
\text { All races } \\
\text { Rate }\end{array}$ & $\begin{array}{c}\text { Male } \\
\text { All races } \\
\text { SE }\end{array}$ & $\begin{array}{c}\text { Male } \\
\text { White } \\
\text { Rate }\end{array}$ & $\begin{array}{c}\text { Male } \\
\text { White } \\
\text { SE }\end{array}$ & $\begin{array}{l}\text { Male } \\
\text { Black } \\
\text { Rate }\end{array}$ & $\begin{array}{c}\text { Male } \\
\text { Black } \\
\text { SE }\end{array}$ & $\begin{array}{c}\text { Female } \\
\text { All races } \\
\text { Rate }\end{array}$ & $\begin{array}{c}\text { Female } \\
\text { All races } \\
\text { SE }\end{array}$ & $\begin{array}{c}\text { Female } \\
\text { White } \\
\text { Rate }\end{array}$ & $\begin{array}{c}\text { Female } \\
\text { White } \\
\text { SE }\end{array}$ & $\begin{array}{c}\text { Female } \\
\text { Black } \\
\text { Rate }\end{array}$ & $\begin{array}{c}\text { Female } \\
\text { Black } \\
\text { SE }\end{array}$ \\
\hline \multicolumn{13}{|l|}{$\begin{array}{l}\text { Esophageal } \\
\text { cancer }\end{array}$} \\
\hline 1st quintile & 11.54 & 0.35 & 7.51 & 0.37 & 22.04 & 0.90 & 3.47 & 0.17 & 2.21 & 0.17 & 6.44 & 0.41 \\
\hline 2nd quintile & 7.17 & 0.25 & 6.72 & 0.26 & 13.25 & 1.25 & 1.82 & 0.11 & 1.71 & 0.11 & 3.17 & 0.48 \\
\hline 3rd quintile & 6.94 & 0.23 & 6.68 & 0.25 & 11.99 & 1.61 & 2.04 & 0.11 & 2.05 & 0.11 & 3.71 & 0.74 \\
\hline 4th quintile & 6.68 & 0.23 & 6.72 & 0.24 & 8.93 & 1.70 & 2.01 & 0.11 & 2.03 & 0.11 & 2.50 & 0.71 \\
\hline 5th quintile & 5.49 & 0.21 & 5.39 & 0.22 & 8.50 & 2.20 & 2.13 & 0.11 & 2.21 & 0.12 & 4.06 & 1.41 \\
\hline RR (Q1/Q5) & $2.10^{*}$ & & $1.39^{*}$ & & $2.59^{*}$ & & $1.63^{*}$ & & 1.00 & & 1.59 & \\
\hline$P_{\text {trend }}$ & $<0.01$ & & $<0.01$ & & $<0.01$ & & $>0.05$ & & $>0.05$ & & $>0.05$ & \\
\hline $\begin{array}{l}\text { Breast cancer } \\
\text { (female) }\end{array}$ & & & \multicolumn{10}{|c|}{ Cervical cancer } \\
\hline 1st quintile & 101.38 & 0.90 & 103.29 & 1.20 & 110.96 & 1.70 & 19.71 & 0.40 & 21.47 & 0.55 & 18.51 & 0.68 \\
\hline 2nd quintile & 119.20 & 0.89 & 122.64 & 1.00 & 116.61 & 2.92 & 12.13 & 0.29 & 12.07 & 0.32 & 13.34 & 0.98 \\
\hline 3rd quintile & 127.43 & 0.87 & 130.31 & 0.95 & 123.28 & 3.95 & 10.50 & 0.25 & 9.91 & 0.27 & 14.62 & 1.31 \\
\hline 4th quintile & 134.09 & 0.88 & 137.23 & 0.94 & 124.95 & 5.20 & 8.55 & 0.22 & 8.31 & 0.23 & 11.53 & 1.47 \\
\hline 5th quintile & 148.53 & 0.92 & 152.04 & 0.98 & 134.45 & 6.69 & 7.41 & 0.20 & 7.00 & 0.21 & 14.22 & 2.09 \\
\hline RR (Q1/Q5) & $0.68^{*}$ & & $0.68^{*}$ & & $0.83^{*}$ & & $2.66^{*}$ & & $3.07^{*}$ & & 1.30 & \\
\hline$P_{\text {trend }}$ & $<0.01$ & & $<0.01$ & & $<0.01$ & & $<0.01$ & & $<0.01$ & & $>0.05$ & \\
\hline \multicolumn{13}{|c|}{ Prostate cancer } \\
\hline 1st quintile & 154.11 & 1.34 & 133.67 & 1.61 & 229.51 & 3.12 & & & & & & \\
\hline 2nd quintile & 164.11 & 1.19 & 164.57 & 1.29 & 240.49 & 5.86 & & & & & & \\
\hline 3rd quintile & 178.61 & 1.22 & 181.46 & 1.31 & 261.93 & 8.41 & & & & & & \\
\hline 4th quintile & 179.84 & 1.24 & 181.05 & 1.29 & 254.85 & 10.87 & & & & & & \\
\hline 5th quintile & 195.63 & 1.32 & 197.32 & 1.37 & 280.15 & 13.76 & & & & & & \\
\hline RR (Q1/Q5) & $0.79^{*}$ & & $0.68^{*}$ & & $0.82^{*}$ & & & & & & & \\
\hline$P_{\text {trend }}$ & $<0.01$ & & $<0.01$ & & $<0.01$ & & & & & & & \\
\hline
\end{tabular}

Incidence rates are age-adjusted to the $2000 \mathrm{US}$ standard population. $\mathrm{RR}=$ rate ratio (SES quintile $1 / \mathrm{SES}$ quintile 5 ). ${ }^{*} P<0.05$.

Quintile 1 represents low SES and high deprivation level, whereas quintile 5 denotes high SES and low deprivation level.

The 11 SEER registries include the states of Connecticut, Hawaii, Iowa, New Mexico, and Utah; and the metropolitan areas of Atlanta, Detroit, Los Angeles, San Francisco and Oakland, San Jose and Monterey, and Seattle.

prostate, breast, and cervical cancer. Low-SES individuals and residents of more deprived neighborhoods have substantially higher rates of late-stage diagnoses of lung, colorectal, prostate, breast, and cervical cancer and significantly lower rates of cancer survival than their counterparts from more affluent neighborhoods or SES backgrounds $[6,10,16,24,31-$ 37]. Lack of health insurance, limited access to care, and lower rates of regular pap smear, mammography, and colorectal cancer screening among lower SES individuals (as shown in Table 5) and among residents of more disadvantaged areas may account for their higher rates of late-stage cancer diagnoses [2, 3, 6, 33-35]. However, lower cancer survival rates among the disadvantaged may reflect their not only higher rates of late-stage cancer diagnoses, but also less favorable cancer treatment or medical care $[3,6,35]$.

Research suggests that SES and area deprivation levels do not fully account for racial/ethnic disparities in cancer incidence, mortality, and outcomes in the US $[3,6,10,11,14]$.
For example, within each deprivation group, Blacks have higher all-cancer mortality rates than Whites. Indeed, the overall cancer mortality and incidence rates for Blacks in the most-affluent group are similar to or exceed those for Whites in the most-deprived group $[3,6]$. Within each SES or deprivation group, Black women have approximately two times higher cervical cancer mortality and $50 \%$ higher breast cancer mortality than White women $[3,6]$. Black men in each deprivation group have at least two times higher prostate cancer mortality rates than their White counterparts $[3,6]$. Such marked racial inequalities may exist partly because Blacks are socially and materially worse off than Whites across different socioeconomic strata $[2,3]$. Moreover, they are more likely to be disadvantaged than Whites in health-risk behaviors, healthcare access and use, and cancer treatment and survival within each deprivation group $[2,3,6]$.

Detection of cancer at an early, localized stage may be considered a marker for access to healthcare and preventive 

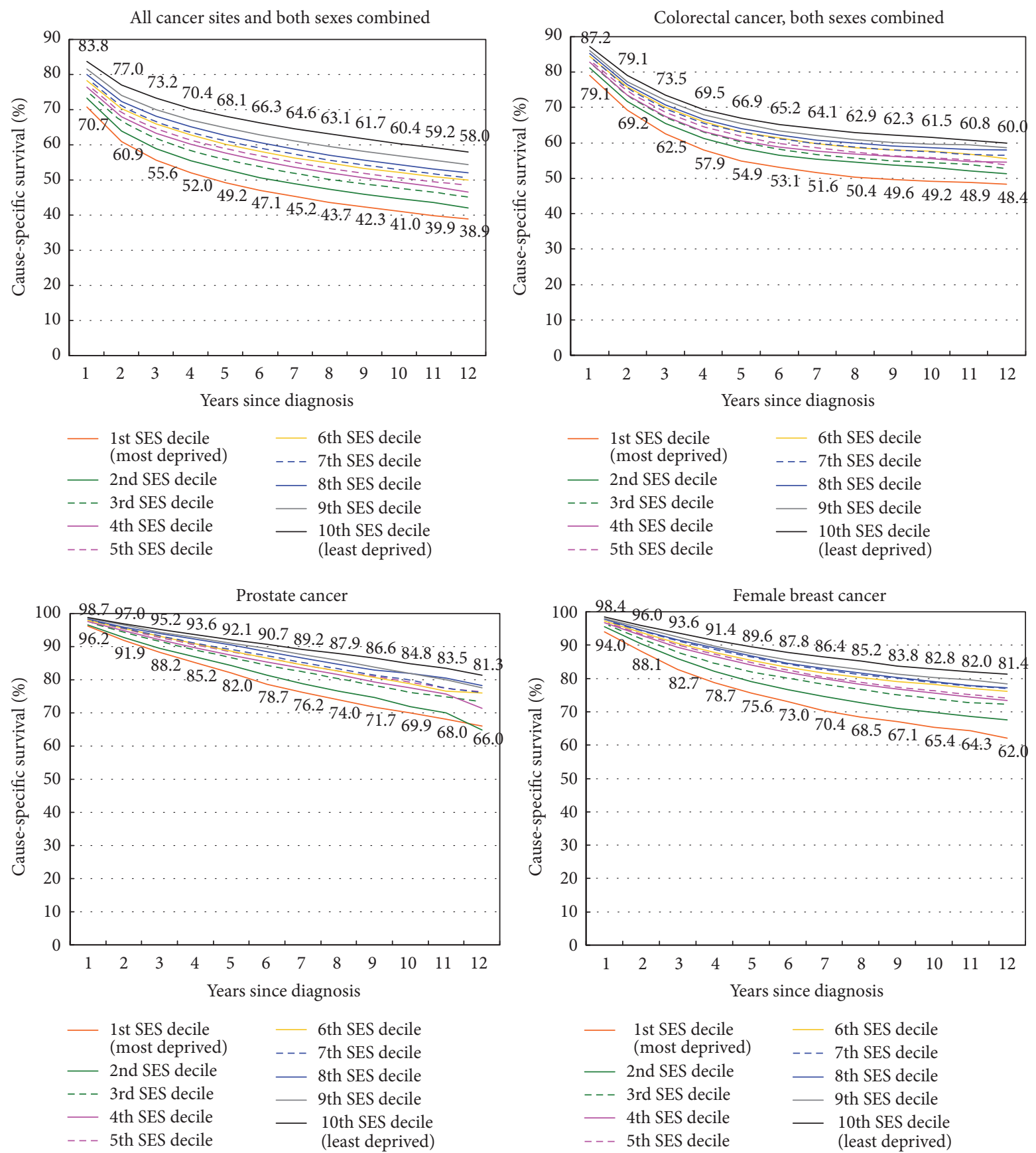

FIGURE 6: Cancer survival by census tract socioeconomic deprivation index, 11 SEER registries, United States, 1988-1999.

health services, including cancer screening $[6,26]$. Studies have shown significant Black-White and socioeconomic disparities in stage at cancer diagnosis $[6,10]$. Within each SES or deprivation group, Blacks have a higher likelihood than Whites of being diagnosed with advanced-stage colorectal, prostate, breast, and cervical cancers $[6,10]$. Additionally, even after controlling for stage at diagnosis, Blacks, in each deprivation group, have significantly lower survival rates from colorectal, prostate, breast, and cervical cancer than Whites $[6,10,38-40]$.
4.1. Comparison with International Patterns. Although studies of cancer inequalities vary widely in their use of socioeconomic measures and coverage of time periods, socioeconomic disparities in US cancer mortality, incidence, and survival reported here are generally consistent with patterns observed for the other industrialized countries $[3,12,15]$. Consistent with the US pattern, all-cancer mortality rates in England during the past decade increased consistently by area deprivation levels [41, 42]. In several European populations, cancer mortality rates were significantly higher among both 
TABLE 4: Multivariate Cox regression models showing adjusted ${ }^{1}$ relative risks (hazard ratios) of mortality among cancer patients diagnosed during 1988-1999, 11 SEER registries (maximum mortality follow-up of 11 years).

\begin{tabular}{|c|c|c|c|c|c|c|c|c|c|c|c|c|}
\hline \multirow[b]{3}{*}{ Race/ethnicity } & \multicolumn{3}{|c|}{$\begin{array}{l}\text { All cancer sites and both } \\
\text { sexes combined }\end{array}$} & \multicolumn{3}{|c|}{$\begin{array}{l}\text { Colorectal cancer both } \\
\text { sexes combined }\end{array}$} & \multicolumn{3}{|c|}{ Prostate cancer ${ }^{4}$} & \multicolumn{3}{|c|}{ Female breast cancer ${ }^{5}$} \\
\hline & \multirow[t]{2}{*}{$\begin{array}{l}\text { Hazard } \\
\text { ratio }\end{array}$} & \multicolumn{2}{|c|}{$\begin{array}{l}\text { 95\% confidence } \\
\text { interval }\end{array}$} & \multirow[t]{2}{*}{$\begin{array}{l}\text { Hazard } \\
\text { ratio }\end{array}$} & \multicolumn{2}{|c|}{$\begin{array}{l}95 \% \text { confidence } \\
\text { interval }\end{array}$} & \multirow[t]{2}{*}{$\begin{array}{l}\text { Hazard } \\
\text { ratio }\end{array}$} & \multicolumn{2}{|c|}{$\begin{array}{l}\text { 95\% confidence } \\
\text { interval }\end{array}$} & \multirow[t]{2}{*}{$\begin{array}{l}\text { Hazard } \\
\text { ratio }\end{array}$} & \multicolumn{2}{|c|}{$\begin{array}{c}95 \% \text { confidence } \\
\text { interval }\end{array}$} \\
\hline & & & & & \multirow{2}{*}{\multicolumn{2}{|c|}{ Reference }} & & \multirow{2}{*}{\multicolumn{2}{|c|}{ Reference }} & & & \\
\hline $\begin{array}{l}\text { Non-Hispanic } \\
\text { White }\end{array}$ & 1.00 & \multicolumn{2}{|c|}{ Reference } & 1.00 & & & 1.00 & & & \multirow{2}{*}{$\begin{array}{l}1.00 \\
1.60\end{array}$} & \multicolumn{2}{|c|}{ Reference } \\
\hline $\begin{array}{l}\text { Non-Hispanic } \\
\text { Black }\end{array}$ & 1.20 & 1.19 & 1.21 & 1.18 & 1.14 & 1.23 & 1.43 & 1.37 & 1.50 & & 1.53 & 1.67 \\
\hline Hispanic/Latino & 1.05 & 1.03 & 1.06 & 1.09 & 1.04 & 1.13 & 1.16 & 1.09 & 1.24 & 1.16 & 1.10 & 1.23 \\
\hline American Indian & 1.46 & 1.40 & 1.54 & 1.40 & 1.18 & 1.67 & 1.85 & 1.48 & 2.31 & 1.52 & 1.22 & 1.90 \\
\hline Chinese & 1.22 & 1.20 & 1.25 & 0.92 & 0.86 & 0.99 & 0.86 & 0.74 & 1.00 & 0.91 & 0.80 & 1.04 \\
\hline Japanese & 0.97 & 0.94 & 0.99 & 0.80 & 0.75 & 0.85 & 0.72 & 0.64 & 0.82 & 0.60 & 0.53 & 0.69 \\
\hline Filipino & 1.04 & 1.01 & 1.06 & 0.97 & 0.88 & 1.05 & 1.01 & 0.90 & 1.13 & 1.03 & 0.92 & 1.15 \\
\hline Hawaiian & 1.40 & 1.34 & 1.46 & 1.17 & 1.01 & 1.37 & 1.67 & 1.31 & 2.11 & 1.16 & 0.97 & 1.39 \\
\hline Korean & 1.54 & 1.48 & 1.60 & 0.91 & 0.78 & 1.07 & 1.19 & 0.82 & 1.74 & 1.00 & 0.75 & 1.32 \\
\hline Asian Indian & 0.90 & 0.83 & 0.98 & 0.58 & 0.39 & 0.86 & 1.47 & 1.03 & 2.09 & 1.17 & 0.85 & 1.61 \\
\hline Vietnamese & 1.47 & 1.41 & 1.54 & 0.80 & 0.64 & 1.00 & 1.71 & 1.14 & 2.55 & 1.11 & 0.83 & 1.50 \\
\hline Other API & 1.14 & 1.09 & 1.18 & 0.81 & 0.68 & 0.97 & 1.15 & 0.89 & 1.49 & 1.12 & 0.91 & 1.38 \\
\hline \multicolumn{13}{|l|}{$\begin{array}{l}\text { Neighborhood } \\
\text { (census tract) SES } \\
\text { index }\end{array}$} \\
\hline 1st decile (low SES) & 1.56 & 1.54 & 1.59 & 1.29 & 1.23 & 1.36 & 1.57 & 1.46 & 1.68 & 1.68 & 1.57 & 1.79 \\
\hline 2nd decile & 1.47 & 1.46 & 1.49 & 1.21 & 1.16 & 1.27 & 1.53 & 1.43 & 1.63 & 1.59 & 1.49 & 1.69 \\
\hline 3rd decile & 1.39 & 1.37 & 1.41 & 1.19 & 1.14 & 1.24 & 1.38 & 1.30 & 1.46 & 1.49 & 1.41 & 1.58 \\
\hline 4th decile & 1.34 & 1.33 & 1.36 & 1.17 & 1.12 & 1.22 & 1.28 & 1.21 & 1.36 & 1.40 & 1.32 & 1.48 \\
\hline 5 th decile & 1.31 & 1.29 & 1.32 & 1.15 & 1.11 & 1.20 & 1.26 & 1.19 & 1.33 & 1.35 & 1.28 & 1.42 \\
\hline 6th decile & 1.26 & 1.25 & 1.28 & 1.10 & 1.06 & 1.15 & 1.26 & 1.19 & 1.34 & 1.27 & 1.20 & 1.34 \\
\hline 7th decile & 1.23 & 1.21 & 1.24 & 1.10 & 1.06 & 1.15 & 1.25 & 1.18 & 1.33 & 1.21 & 1.14 & 1.27 \\
\hline 8th decile & 1.20 & 1.18 & 1.21 & 1.08 & 1.04 & 1.13 & 1.18 & 1.11 & 1.26 & 1.19 & 1.13 & 1.26 \\
\hline 9th decile & 1.12 & 1.11 & 1.13 & 1.05 & 1.01 & 1.10 & 1.11 & 1.04 & 1.18 & 1.14 & 1.07 & 1.20 \\
\hline $\begin{array}{l}\text { 10th decile (high } \\
\text { SES) }\end{array}$ & 1.00 & \multicolumn{2}{|c|}{ Reference } & 1.00 & \multicolumn{2}{|c|}{ Reference } & 1.00 & \multicolumn{2}{|c|}{ Reference } & 1.00 & \multicolumn{2}{|c|}{ Reference } \\
\hline
\end{tabular}

${ }^{1}$ Adjusted for age at diagnosis, period of diagnosis, sex, race/ethnicity, marital status, area SES, and rural-urban residence.

${ }^{2}$ Number of diagnosed cancer patients $=1,663,844$; number dying during 1988-1999 follow-up $=541,427$.

${ }^{3}$ Number of diagnosed colorectal cancer patients = 150,330; number dying during 1988-1999 follow-up = 46,673.

${ }^{4}$ Number of diagnosed prostate cancer patients = 228,839; number dying during 1988-1999 follow-up = 422,784.

${ }^{5}$ Number of diagnosed female breast cancer patients = 197,270; number dying during 1988-1999 follow-up = 24,976.

males and females in lower education groups [12]. Consistent with the US pattern, lung cancer mortality rates in Canada increased in relation to deprivation levels [43]. Higher lung cancer mortality rates were found among men in lower SES groups in many European countries [12, 44]. Inverse socioeconomic gradients in US colorectal cancer mortality rates are compatible with occupational and educational patterns in mortality observed among several European countries [12,45]. Marked socioeconomic disparities in US cervical cancer mortality reported here are generally consistent with those shown for other industrialized countries. An approximately twofold higher cervical cancer mortality was found among women in low- than high-SES groups in a study that compared inequalities in various low/middle income countries, North America, and Europe, although the magnitude of socioeconomic inequalities was greater in North America than in Europe [43, 46, 47]. Consistent with the US pattern, cancer survival rates have decreased consistently by deprivation levels in the UK, Australia, and New Zealand [36, 48, 49].

4.2. Limitations. This study has some limitations. The SES or deprivation indices used in the mortality trend analysis were defined at the county-level, which could vary greatly across census tracts or neighborhoods within a given county. Unfortunately, census tract geocodes are not available in 


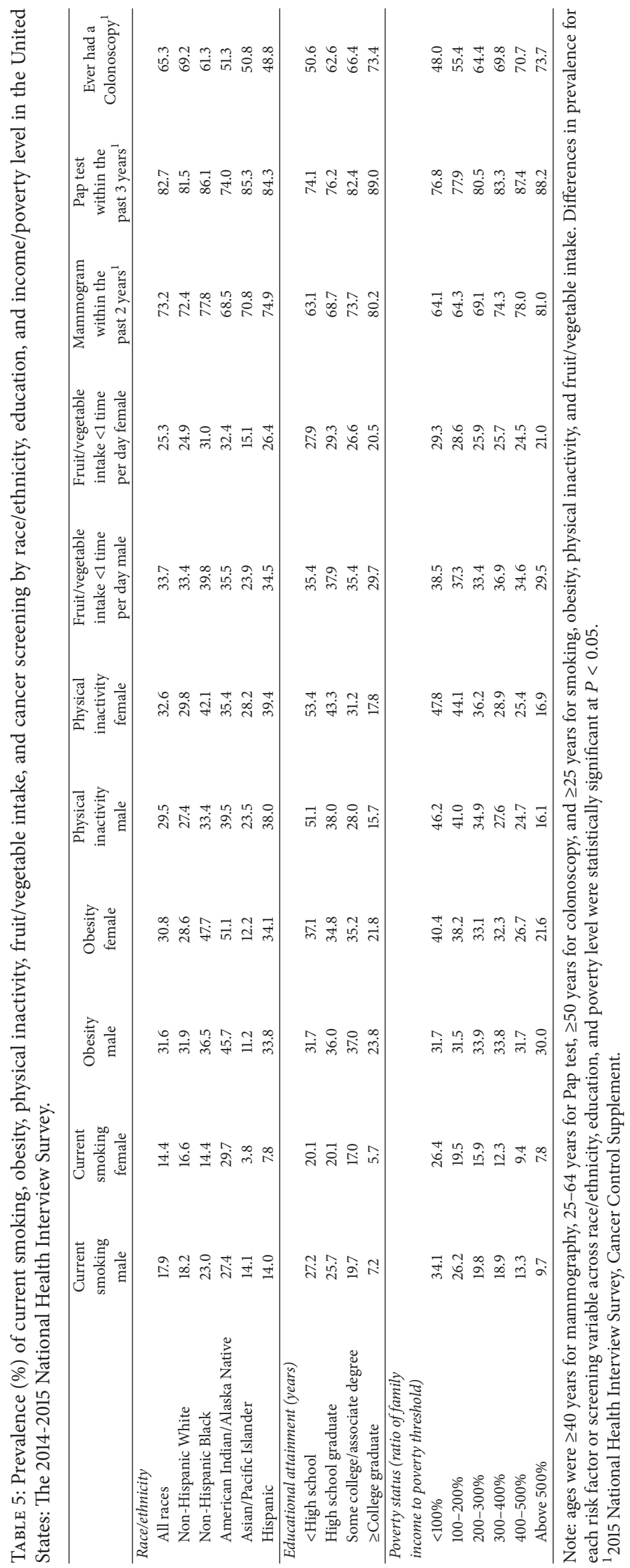


the national mortality database for confidentiality protection of individual information on the death certificate, and the linkage of US mortality records and area-based SES measures is not feasible at the neighborhood level [3-6, 27, $50,51]$. Given the compositional heterogeneity of counties, the association between deprivation and cancer mortality is likely to be underestimated [3-6, 50, 51]. Additionally, the use of 1970 and 1990 census-based deprivation indices to characterize cancer mortality trends from 1950 through 1998 might have influenced SES-specific mortality patterns if socioeconomic position of some counties changed over time. However, previous analyses using county deprivation indices for different censuses/time periods have demonstrated temporal stability of the SES/deprivation index in terms of its relative socioeconomic classification of US counties over time. These analyses have indicated a high degree of correspondence (correlation >0.90) between 1970, 1980, and 1990 SES indices and essentially similar long-term trends in area socioeconomic inequalities in mortality whether trends were based on the 1970, 1980, or 1990 indices for the entire study period [50, 51]. Our analysis indicates correlations among the 1990, 2000, and 2008-2012 deprivation indices to be $>0.95$. The high correlations among the indices and the relatively stable socioeconomic standing of county deprivation groups indicate that the broad geographical distribution of deprivation and socioeconomic disadvantage in the United States has changed very little over the past several decades $[50,51]$. The small degree of area misclassification that may arise from using different SES indices during the study period is unlikely to affect the general trend of changing cancer mortality patterns shown here.

Another limitation concerns conducting a large number of statistical tests without correcting for multiple comparisons. Most of the statistical tests in this study were conducted using the 0.05 level of significance, implying that $5 \%$ of the tests could have been significant by chance. Lowering the alpha cutoff to 0.01 may address this problem to a large extent; indeed, the availability of mortality and incidence rates along with their standard errors in Tables 1-3 allows one to compute and evaluate $t$-tests for significance at the 0.01 level. However, lowering the Type 1 error rate by decreasing the alpha level to 0.01 or 0.001 level comes at the cost of increasing Type II error (failing to detect a difference when there really is one).

4.3. Conclusions. Cancer is the second leading cause of death in the US and the most prominent cause of death in terms of years of potential life lost [2, 3, 21]. Evidence presented here indicates how cancer disparities contribute greatly to the overall health inequalities in the US. With large socioeconomic and racial/ethnic inequalities in smoking, obesity, and physical inactivity among young people continuing to persist, inequalities in US cancer mortality and incidence are not expected to diminish in the foreseeable future [2, 3]. Efforts to reduce cancer disparities, especially those in lung cancer, therefore might include tobacco control policies at the national and local levels that place greater smoking restrictions or legislate against smoking in public places, ban tobacco marketing, reduce tobacco availability, increase financial and other barriers to smoking, and provide targeted smoking cessation programs for those in socially disadvantaged groups or areas $[3,6]$. Healthcare inequalities have also risen in both absolute and relative terms and socioeconomic and racial/ethnic disparities in stage at diagnosis and survival from major cancers have persisted $[3,6]$. These trends would also imply continued social inequalities in cancer mortality and incidence in the future. Health policies therefore should enhance access to cancer screening programs among the disadvantaged populations and underserved areas. Lastly, social policy measures aimed at improving the broader social determinants, such as material living conditions and the social and physical environments, are needed to tackle health inequalities in cancer outcomes $[3,6]$.

\section{Disclosure}

The views expressed are the authors' and not necessarily those of the Health Resources and Services Administration or the US Department of Health and Human Services.

\section{Conflicts of Interest}

The authors declare that there are no conflicts of interest.

\section{References}

[1] US Department of Health and Human Services, Healthy People 2020, http://www.healthypeople.gov/2020/default.aspx.

[2] National Center for Health Statistics, Health, United States, 2011 with Special Feature on Socioeconomic Status and Health, US Department of Health and Human Services, Hyattsville, Md, USA, 2012.

[3] G. K. Singh, S. D. Williams, M. Siahpush, and A. Mulhollen, "Socioeconomic, rural-urban, and racial inequalities in US cancer mortality: Part I-all cancers and lung cancer and part II-colorectal, prostate, breast, and cervical cancers," Journal of Cancer Epidemiology, vol. 2011, Article ID 107497, 27 pages, 2011.

[4] G. K. Singh, B. A. Miller, B. F. Hankey, E. J. Feuer, and L. W. Pickle, "Changing area socioeconomic pattern in U.S. cancer mortality, 1950-1998: Part I-all cancers among men," Journal of the National Cancer Institute, vol. 94, no. 12, pp. 904-915, 2002.

[5] G. K. Singh, B. A. Miller, and B. F. Hankey, "Changing area socioeconomic patterns in U.S. cancer mortality, 1950-1998: Part II-lung and colorectal cancers," Journal of the National Cancer Institute, vol. 94, no. 12, pp. 916-925, 2002.

[6] G. K. Singh, B. A. Miller, B. F. Hankey, and B. K. Edwards, Area Socioeconomic Variations in U.S. Cancer Incidence, Mortality, Stage, Treatment, and Survival, 1975-1999, NCI Cancer Surveillance Monograph Series No. 4, NIH Publication No. 03-5417, National Cancer Institute, Bethesda, Md, USA, 2003, http://seer.cancer.gov/publications/ses/index.html.

[7] G. K. Singh and A. Jemal, "Socioeconomic inequalities in cancer incidence and mortality," in American Cancer Society's Clinical Oncology Textbook, T. Gansler, Ed., Wiley, New York, NY, USA, 2017.

[8] F. Faggiano, T. Partanen, M. Kogevinas, and P. Boffetta, "Socioeconomic differences in cancer incidence and mortality," IARC Scientific Publications, no. 138, pp. 65-176, 1997.

[9] G. D. Smith, D. Leon, M. J. Shipley, and G. Rose, "Socioeconomic differentials in cancer among men," International Journal of Epidemiology, vol. 20, no. 2, pp. 339-345, 1991. 
[10] G. K. Singh, B. A. Miller, B. F. Hankey, and B. K. Edwards, "Persistent area socioeconomic disparities in U.S. incidence of cervical cancer, mortality, stage, and survival, 1975-2000," Cancer, vol. 101, no. 5, pp. 1051-1057, 2004.

[11] G. K. Singh and M. Siahpush, "All-cause and cause-specific mortality of immigrants and native born in the United States," American Journal of Public Health, vol. 91, no. 3, pp. 392-399, 2001.

[12] G. Menvielle, A. E. Kunst, I. Stirbu et al., "Educational differences in cancer mortality among women and men: a gender pattern that differs across Europe," British Journal of Cancer, vol. 98, no. 5, pp. 1012-1019, 2008.

[13] K. Steenland, J. Henley, and M. Thun, "All-cause and causespecific death rates by educational status for two million people in two American Cancer Society cohorts, 1959-1996," American Journal of Epidemiology, vol. 156, no. 1, pp. 11-21, 2002.

[14] K. C. Chu, B. A. Miller, and S. A. Springfield, "Measures of racial/ethnic health disparities in cancer mortality rates and the influence of socioeconomic status," Journal of the National Medical Association, vol. 99, no. 10, pp. 1092-1104, 2007.

[15] M. Kogevinas, N. Pearce, M. Susser, and P. Boffetta, "Social inequalities and cancer," IARC Scientific Publications 138, 1997.

[16] L. X. Clegg, M. E. Reichman, B. A. Miller et al., "Impact of socioeconomic status on cancer incidence and stage at diagnosis: selected findings from the surveillance, epidemiology, and end results: National Longitudinal Mortality Study," Cancer Causes and Control, vol. 20, no. 4, pp. 417-435, 2009.

[17] G. K. Singh, M. Siahpush, and S. D. Williams, "Changing urbanization patterns in US lung cancer mortality, 1950-2007," Journal of Community Health, vol. 37, no. 2, pp. 412-420, 2012.

[18] G. K. Singh, M. Siahpush, and S. F. Altekruse, "Time trends in liver cancer mortality, incidence, and risk factors by unemployment level and race/ethnicity, United States, 1969-2011," Journal of Community Health, vol. 38, no. 5, pp. 926-940, 2013.

[19] G. K. Singh, M. Siahpush, R. A. Hiatt, and L. R. Timsina, "Dramatic increases in obesity and overweight prevalence and body mass index among ethnic-immigrant and social class groups in the United States, 1976-2008," Journal of Community Health, vol. 36, no. 1, pp. 94-110, 2011.

[20] D. L. Blackwell, J. W. Lucas, and T. C. Clarke, "Summary health statistics for U.S. adults: national health interview survey, 2012," Vital and Health Statistics, vol. 10, no. 260, pp. 1-161, 2014.

[21] K. D. Kochanek, S. L. Murphy, J. Q. Xu, and B. Tejada-Vera, "Deaths: final data for 2014," National Vital Statistics Reports, vol. 65 , no. 4, pp. 1-121, 2016.

[22] N. Howlader, A. M. Noone, M. Krapcho et al., Eds., SEER Cancer Statistics Review, 1975-2013, National Cancer Institute, Bethesda, Md, USA, 2016, http://seer.cancer.gov/csr/1975_2013/.

[23] US Census Bureau, National Longitudinal Mortality Study, Reference Manual, US Census Bureau, Washington, DC, USA, 2012, http://www.census.gov/did/www/nlms/publications/reference.html.

[24] X. L. Du, C. C. Lin, N. J. Johnson, and S. Altekruse, "Effects of individual-level socioeconomic factors on racial disparities in cancer treatment and survival," Cancer, vol. 117, no. 14, pp. 32423251, 2011.

[25] A. Jemal, E. P. Simard, C. Dorell et al., "Annual report to the nation on the status of cancer, 1975-2009, featuring the burden and trends in human papillomavirus (HPV)-associated cancers and HPV vaccination coverage levels," Journal of the National Cancer Institute, vol. 105, no. 3, pp. 175-201, 2013.
[26] American Cancer Society, Cancer Facts \& Figures 2016, American Cancer Society, Atlanta, Ga, USA, 2016.

[27] G. K. Singh, "Area deprivation and widening inequalities in US mortality, 1969-1998," American Journal of Public Health, vol. 93, no. 7, pp. 1137-1143, 2003.

[28] US Census Bureau, The American Community Survey, US Census Bureau, Washington, DC, USA, 2016, http://www.census .gov/acs/www/.

[29] National Center for Health Statistics, The National Health Interview Survey, Questionnaires, Datasets, and Related Documentation: 2014-2015 Public Use Data Files, US Department of Health and Human Services, Hyattsville, Md, USA, 2015, http://www.cdc.gov/nchs/nhis/nhis_questionnaires.htm.

[30] S. H. Rim, D. A. Joseph, C. B. Steele, T. D. Thompson, and L. C. Seeff, "Colorectal cancer screening-United States, 2002, 2004, 2006, and 2008," Morbidity and Mortality Weekly Report, vol. 60, pp. 42-46, 2011.

[31] J. Mandelblatt, H. Andrews, J. Kerner, A. Zauber, and W. Burnett, "Determinants of late stage diagnosis of breast and cervical cancer: the impact of age, race, social class, and hospital type," American Journal of Public Health, vol. 81, no. 5, pp. 646649, 1991.

[32] A. Parikh-Patel, J. H. Bates, and S. Campleman, "Colorectal cancer stage at diagnosis by socioeconomic and urban/rural status in California, 1988-2000," Cancer, vol. 107, pp. 1189-1195, 2006.

[33] P. D. Baade, G. Turrell, and J. F. Aitken, "Geographic remoteness, area-level socio-economic disadvantage and advanced breast cancer: a cross-sectional, multilevel study," Journal of Epidemiology and Community Health, vol. 65, no. 11, pp. 10371043, 2011.

[34] M. Kogevinas and M. Porta, "Socioeconomic differences in cancer survival: a review of the evidence," IARC Scientific Publications, no. 138, pp. 177-206, 1997.

[35] A. Auvinen and S. Karjalainen, "Possible explanations for social class differences in cancer patient survival," IARC scientific publications, no. 138, pp. 377-397, 1997.

[36] B. Rachet, L. Ellis, C. Maringe et al., "Socioeconomic inequalities in cancer survival in England after the NHS cancer plan," British Journal of Cancer, vol. 103, no. 4, pp. 446-453, 2010.

[37] C. J. Bradley, C. W. Given, and C. Roberts, "Race, socioeconomic status, and breast cancer treatment and survival," Journal of the National Cancer Institute, vol. 94, no. 7, pp. 490-496, 2002.

[38] A. M. McCarthy, T. Dumanovsky, K. Visvanathan, A. R. Kahn, and M. J. Schymura, "Racial/ethnic and socioeconomic disparities in mortality among women diagnosed with cervical cancer in New York City, 1995-2006," Cancer Causes and Control, vol. 21, no. 10, pp. 1645-1655, 2010.

[39] A. S. Robbins, A. S. Whittemore, and D. H. Thom, "Differences in socioeconomic status and survival among white and black men with prostate cancer," American Journal of Epidemiology, vol. 151, no. 4, pp. 409-416, 2000.

[40] S. Marcella and J. E. Miller, "Racial differences in colorectal cancer mortality: the importance of stage and socioeconomic status," Journal of Clinical Epidemiology, vol. 54, no. 4, pp. 359366, 2001.

[41] Department of Health, Tackling Health Inequalities: 2007 Status Report on the Programme for Action, Health Inequalities Unit, Department of Health, London, UK, 2007.

[42] Cancer Research UK and National Cancer Intelligence Network, Cancer. Cancer by Deprivation in England: Incidence, 1996-2010, Mortality, 1997-2011, NCIN, London, UK, 2014. 
[43] R. Wilkins, J. Berthelot, and E. Ng, "Trends in mortality by neighbourhood income in urban Canada from 1971 to 1996," Health Reports, vol. 13, pp. 1-27, 2002.

[44] J. H. A. Van der Heyden, M. M. Schaap, A. E. Kunst et al., "Socioeconomic inequalities in lung cancer mortality in 16 European populations," Lung Cancer, vol. 63, no. 3, pp. 322-330, 2009.

[45] M. J. Aarts, V. E. P. P. Lemmens, M. W. J. Louwman, A. E. Kunst, and J. W. W. Coebergh, "Socioeconomic status and changing inequalities in colorectal cancer? A review of the associations with risk, treatment and outcome," European Journal of Cancer, vol. 46, no. 15, pp. 2681-2695, 2010.

[46] E. Ng, R. Wilkins, M. F. K. Fung, and J.-M. Berthelot, "Cervical cancer mortality by neighbourhood income in urban Canada from 1971 to 1996," Canadian Medical Association Journal, vol. 170, no. 10, pp. 1545-1549, 2004.

[47] S. Parikh, P. Brennan, and P. Boffetta, "Meta-analysis of social inequality and the risk of cervical cancer," International Journal of Cancer, vol. 105, no. 5, pp. 687-691, 2003.

[48] J. F. Stanbury, P. D. Baade, Y. Yu, and X. Q. Yu, "Cancer survival in New South Wales, Australia: socioeconomic disparities remain despite overall improvements," BMC Cancer, vol. 16, no. 1, article no. 48, 2016.

[49] M. Jeffreys, D. Sarfati, V. Stevanovic et al., "Socioeconomic inequalities in cancer survival in New Zealand: the role of extent of disease at diagnosis," Cancer Epidemiology Biomarkers and Prevention, vol. 18, no. 3, pp. 915-921, 2009.

[50] G. K. Singh and M. Siahpush, "Increasing inequalities in allcause and cardiovascular mortality among US adults aged 2564 years by area socioeconomic status, 1969-1998," International Journal of Epidemiology, vol. 31, no. 3, pp. 600-613, 2002.

[51] G. K. Singh and M. D. Kogan, "Widening socioeconomic disparities in US childhood mortality, 1969-2000," American Journal of Public Health, vol. 97, no. 9, pp. 1658-1665, 2007. 


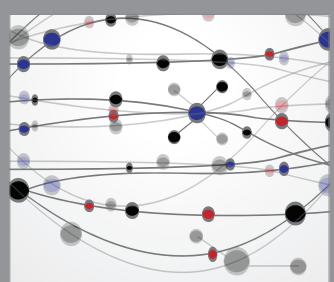

The Scientific World Journal
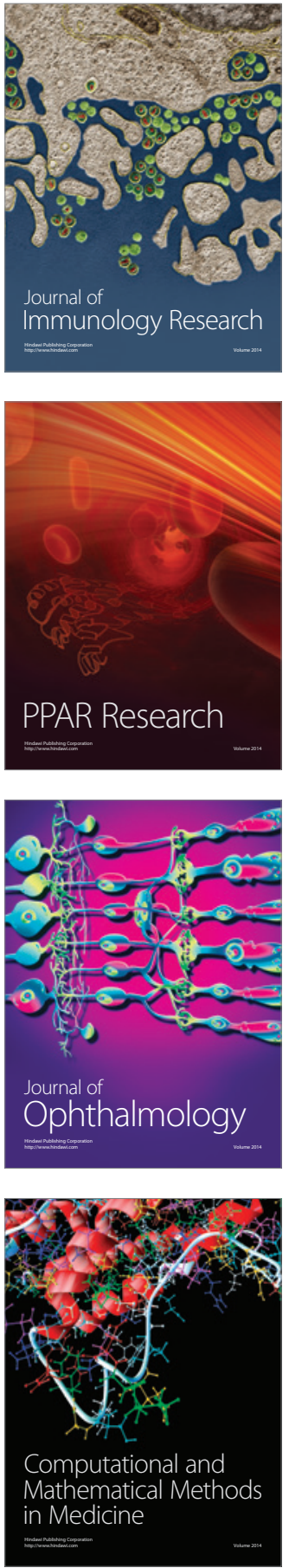

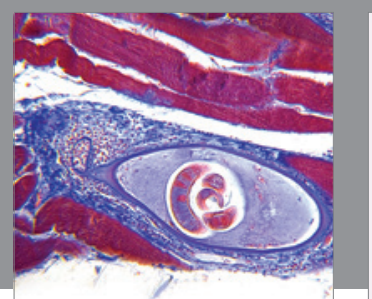

Gastroenterology Research and Practice
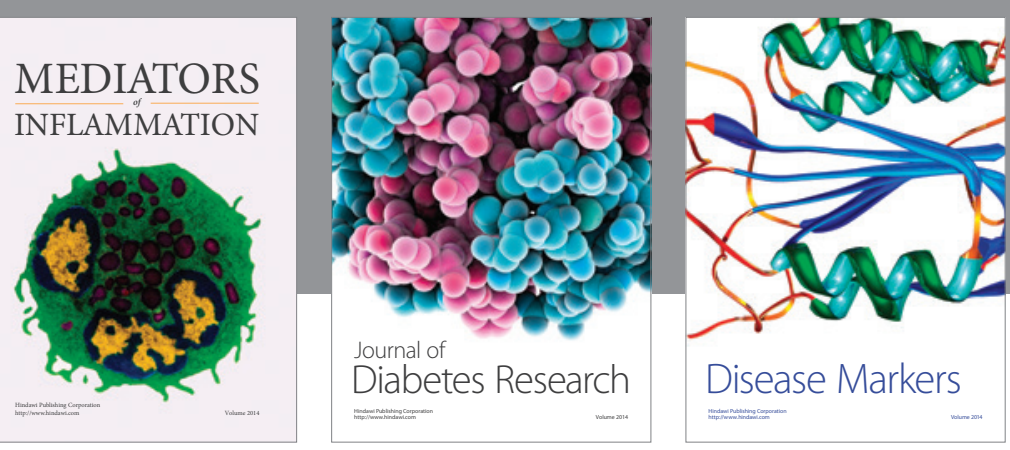

Disease Markers

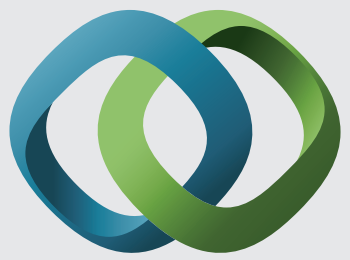

\section{Hindawi}

Submit your manuscripts at

https://www.hindawi.com
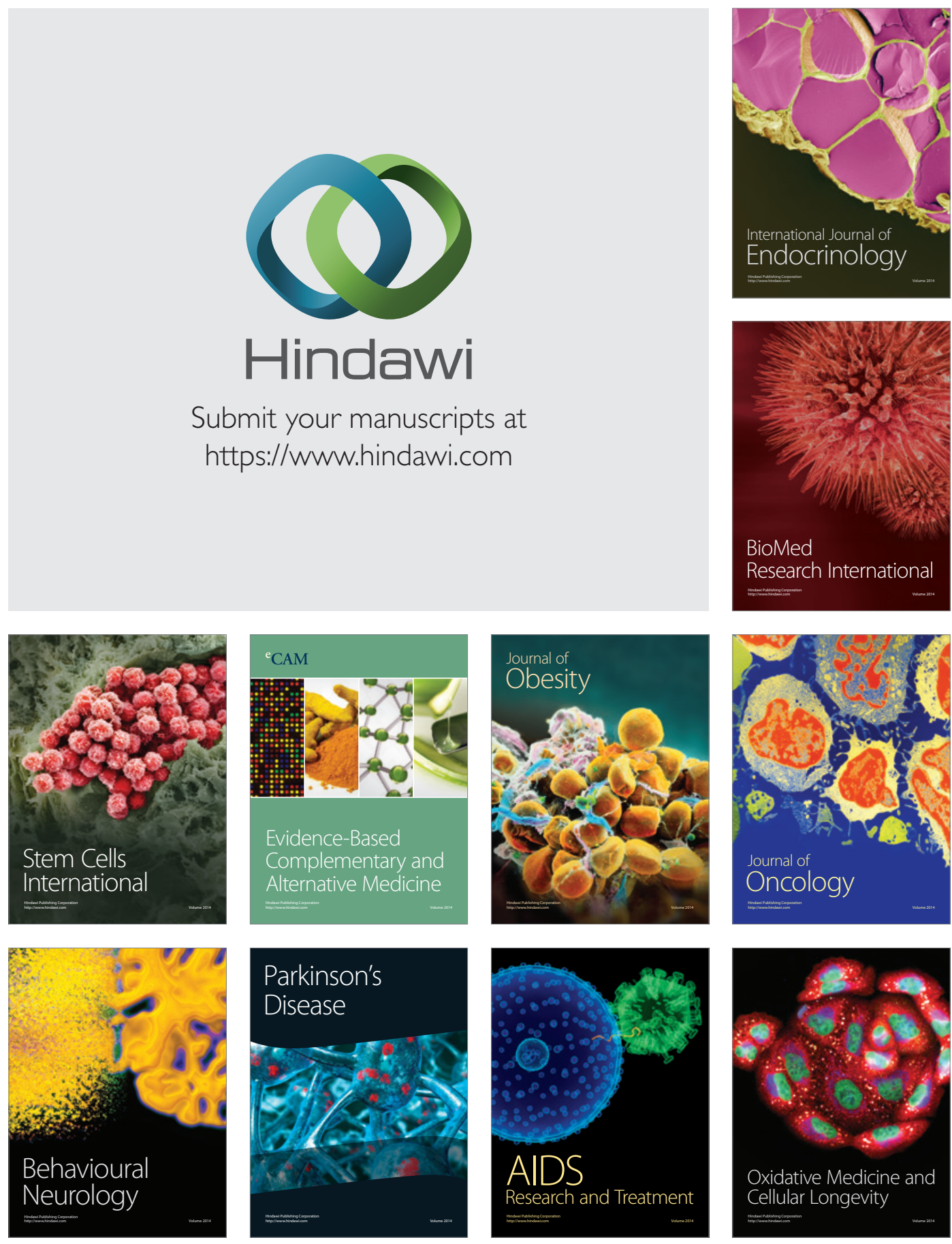